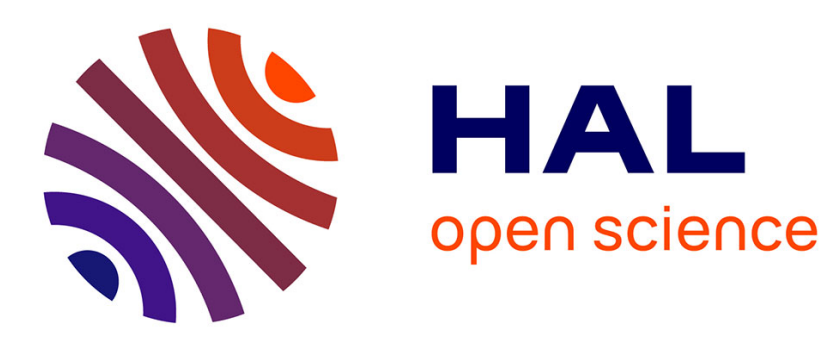

\title{
Necessary Conditions of asymptotic stability for unilateral dynamical systems
}

Daniel Goeleven, Bernard Brogliato

\section{To cite this version:}

Daniel Goeleven, Bernard Brogliato. Necessary Conditions of asymptotic stability for unilateral dynamical systems. [Research Report] RR-5171, INRIA. 2004. inria-00071417

\section{HAL Id: inria-00071417 https://hal.inria.fr/inria-00071417}

Submitted on 23 May 2006

HAL is a multi-disciplinary open access archive for the deposit and dissemination of scientific research documents, whether they are published or not. The documents may come from teaching and research institutions in France or abroad, or from public or private research centers.
L'archive ouverte pluridisciplinaire HAL, est destinée au dépôt et à la diffusion de documents scientifiques de niveau recherche, publiés ou non, émanant des établissements d'enseignement et de recherche français ou étrangers, des laboratoires publics ou privés. 
INSTITUT NATIONAL DE RECHERCHE EN INFORMATIQUE ET EN AUTOMATIQUE

\section{Necessary Conditions of asymptotic stability for unilateral dynamical systems}

Daniel Goeleven — Bernard Brogliato

$\mathbf{N}^{\circ} \mathbf{5 1 7 1}$

Avril 2004

THÈME 4 



\title{
Necessary Conditions of asymptotic stability for unilateral dynamical systems
}

\author{
Daniel Goeleven *, Bernard Brogliato ${ }^{\dagger}$ \\ Thème 4 - Simulation et optimisation \\ de systèmes complexes \\ Projet Bipop \\ Rapport de recherche $\mathrm{n}^{\circ} 5171$ - Avril $2004-51$ pages
}

\begin{abstract}
In this paper, we develop a mathematical tool that can be used to state necessary conditions of asymptotic stability of isolated stationary solutions of a class of unilateral dynamical systems. More precisely, nonlinear evolution variational inequalities are considered. Instability criteria are also given. Applications can be found in mechanics or electrical circuits.
\end{abstract}

Key-words: Variational inequalities, differential inclusions, topological degree, stability theory for unilateral dynamical systems.

* IREMIA, University of La Réunion, Saint-Denis, 97400, France, Daniel.Goeleven@univ-reunion.fr

$\dagger$ INRIA Rhône-Alpes, ZIRST Montbonnot, 655 avenue de l'Europe, 38334 Saint-Ismier, France, Bernard.Brogliato@inrialpes.fr 


\section{Conditions nécéssaires de stabilité asymptotique des dynamiques systèmes unilatéraux}

Résumé : Cet article propose une méthode mathématique permettant de formuler des conditions nécéssaires de stabilité asymptotique du point déquilibre isolé d'une classe de systèmes dynamiques unilatéraux. On considère des inéquations variationnelles dynamiques. Des applications se trouvent dans les circuits électriques ou les systèmes mécaniques.

Mots-clés : Inéquations variationnelles, inclusions différentielles, degré topologique, théorie de la stabilité pour les systèmes dynamiques unilatéraux. 


\section{Introduction}

The stability of stationary solutions of dynamical systems constitutes a very important topic in Applied Mathematics and Engineering. It is well-known that in the case of a large class of nonlinear differential equations, the spectrum of "linearized" operators determines the Lyapunov stability or instability of an equilibrium. This is known as the Lyapunov's linearization method $[27,28,34]$. However, many important problems in engineering (see $[6,10,11,13,14,17,29,33])$ involve inequalities in their mathematical formulation and consequently possess intrinsic nonsmoothness which cannot be removed by change of coordinates, or by feedback. The models corresponding to such problems are called unilateral dynamical systems or non-regular dynamical systems. A large class of unilateral dynamical systems can be represented under the formalism of evolution variational inequalities, see e.g. $[3,13,14]$. The question of stability of stationary solutions of evolution variational inequalities is much more complicated to be investigated, as it is the case in general for hybrid dynamical systems, see e.g. [20, 21].

The research efforts to develop general mathematical approaches to study stability of stationary solutions of evolution variational inequalities are relatively new. It seems that the first contribution acting in this sense was made by Quittner $[23,24,25]$ for a class of parabolic variational inequalities in Hilbert spaces. More recently, Lyapunov approach and KrakovskiiLaSalle invariance theory have been generalized to evolution variational inequalities and corresponding unilateral dynamical systems (see $[1,12,4,7,15,22]$ and the references cited therein). In [12] sufficient conditions for stability of linear evolution variational inequalities have been derived, and we now propose to study necessary conditions.

Our aim in this paper is to state necessary conditions of asymptotic stability for a class of unilateral dynamical systems. More precisely, we consider the problem: Let $\varphi: \mathbb{R}^{n} \rightarrow$ $\mathbb{R} \cup\{+\infty\}$ be a proper, convex and lower semi-continuous function. Let $F: \mathbb{R}^{n} \rightarrow \mathbb{R}^{n}$ be a nonlinear continuous operator. Let $x_{0} \in \mathbb{R}^{n}$ be given. We consider the problem $P\left(x_{0}\right)$ : Find a unique continuous mapping $t \mapsto u(t)$ such that

$$
\begin{gathered}
\frac{d u}{d t} \in L_{\mathrm{loc}}^{\infty}\left(0,+\infty ; \mathbb{R}^{n}\right) ; \\
u(t) \in D(\partial \varphi), \forall t \geq 0 ; \\
\left\langle\frac{d u}{d t}(t)+F(u(t)), v-u(t)\right\rangle+\varphi(v)-\varphi(u(t)) \geq 0, \forall v \in \mathbb{R}^{n}, \text { a.e. } t \geq 0,
\end{gathered}
$$

and

$$
u(0)=x_{0} .
$$

The variational inequality in (3) can also be formulated as the set-valued differential equation:

$$
\frac{d u}{d t}(t)+F(u(t)) \in-\partial \varphi(u(t)), \text { a.e. } t \geq 0,
$$


where $\partial \varphi$ denotes the subdifferential of $\varphi$. It is assumed that $F(0) \in-\partial \varphi(0)$ i.e. the origin 0 of the system is a trivial stationary solution of (5).

If $\varphi \equiv 0$ then (5) reduces to a standard system of differential equations and it is well known (see e.g. [19]) in this case that if 0 is an isolated zero of $F$ and is asymptotically stable, then there exists $\rho_{0}>0$ such that

$$
\operatorname{deg}\left(F, B_{\rho}, 0\right)=1, \forall \rho \in\left(0, \rho_{0}\right],
$$

where $\operatorname{deg}\left(F, B_{\rho}, 0\right)$ denotes the Brouwer degree of $F$ with respect to the open ball $B_{\rho}:=$ $\left\{x \in \mathbb{R}^{n}:\|x\|<\rho\right\}$ and 0 .

Our goal in this paper is to generalize this famous result to the model in (4)-(5). More precisely, let us define the mapping $\Lambda$ as

$$
\Lambda(x):=x-P_{\varphi}(x-F(x))
$$

where $P_{\varphi}:=\left(i d_{\mathbb{R}^{n}}+\partial \varphi\right)^{-1}$ and $i d_{\mathbb{R}^{n}}$ denotes the identity mapping on $\mathbb{R}^{n}$. We will prove that if 0 is an isolated zero of $\Lambda$ and is asymptotically stable, then there exists $\rho_{0}>0$ such that

$$
\operatorname{deg}\left(\Lambda, B_{\rho}, 0\right)=1, \forall \rho \in\left(0, \rho_{0}\right] .
$$

This result constitutes the main result of the paper and is given in Theorem 5 .

In Section 2, we recall the fundamental properties of the Brouwer degree we will use in this paper. In Section 3, we discuss the main properties of the operator $P_{\varphi}$. In Section 4 , we present a concise review of some recent results in stability theory of unilateral dynamical systems. In Section 5, we introduce the Poincaré operator associated to problem (5). In Section 6 , we prove our main result.

In this paper, we develop also several approaches to compute the number $\operatorname{deg}\left(\Lambda, B_{\rho}, 0\right)$. If $\Lambda$ can be easily evaluated then a first approach consist to use some practical results of degree theory to compute $\operatorname{deg}\left(\Lambda, B_{\rho}, 0\right)$ (see Section 6). The approach is of particular interest if $\varphi \equiv \Psi_{\mathbb{R}_{+}^{n}}$, with $\Psi_{\mathbb{R}_{+}^{n}}$ denoting the indicator function of $\mathbb{R}_{+}^{n}$, since in this case:

$$
\Lambda_{i}(x)=\min \left\{x_{i}, F_{i}(x)\right\} .
$$

This case is of particular interest for the study of complementarity dynamical systems [16]. More generally, if $\varphi \equiv \Psi_{K}$ where $K$ denotes a closed convex set, then we develop a second approach (see Section 7) that uses a result of Quittner [25] which reduces the computation of $\operatorname{deg}\left(\Lambda, B_{\rho}, 0\right)$ to the one of $\operatorname{deg}\left(\Lambda_{L}, B_{\rho}, 0\right)$ where $\Lambda_{L}$ is defined by

$$
\Lambda_{L}(x)=x-P_{K_{0}}\left(x-J_{F}(0) x\right),
$$

where $K_{0}$ is the closed convex cone defined by $K_{0}=\cup_{\alpha>0} \alpha K$ and $J_{F}(0)$ denotes the Jacobian matrix of $F$ at 0 .

If $\varphi$ is a convex and continuous function then we develop a third approach (see Section 8 ) by showing that $\operatorname{deg}\left(\Lambda, B_{\rho}, 0\right)$ can be computed by using some appropriate "Lyapunov function". 
The case of linear complementarity problems is discussed in Section 9. Finally, in Section 10, using our main result together with recent invariant results (see [1]), we prove some instability theorems.

Several examples and applications are given so as to illustrate the theoretical results.

Notations: In the sequel the scalar product on $\mathbb{R}^{n}$ is denoted by $\langle\cdot, \cdot\rangle$ (with the associated norm $\|\cdot\|)$. For $r>0$, we set $B_{r}:=\left\{x \in \mathbb{R}^{n}:\|x\|<r\right\}$. Then $\bar{B}_{r}=\left\{x \in \mathbb{R}^{n}:\|x\| \leq r\right\}$ and $\partial B_{r}=\left\{x \in \mathbb{R}^{n}:\|x\|=r\right\}$.

The identity mapping on $\mathbb{R}^{n}$ is denoted by $i d_{\mathbb{R}^{n}}$. Let $K$ be a nonempty closed convex subset of $\mathbb{R}^{n}$. The dual set of $K$ is defined by $K^{*}:=\left\{w \in \mathbb{R}^{n}:\langle w, x\rangle \geq 0, \forall x \in K\right\}$. The recession cone of $K$ is defined by $K_{\infty}:=\cup_{\lambda>0} \frac{1}{\lambda}\left(K-x_{0}\right)$ where $x_{0}$ is any element of $K$. We denote by $\psi_{K}$ the indicator function of $K$, i.e. $\psi_{K}(x)=0$ if $x \in K$ and $\psi_{K}(x)=+\infty$ if $x \notin K$.

For a function $V \in C^{1}\left(\mathbb{R}^{n} ; \mathbb{R}\right)$ we denote by $V^{\prime}(x)$ the gradient of $V$ at $x \in \mathbb{R}^{n}$.

We denote by $d(s, \mathcal{M})$ the distance from a point $s \in \mathbb{R}^{n}$ to a set $\mathcal{M} \subset \mathbb{R}^{n}$, that is $d(s, \mathcal{M}):=$ $\inf _{m \in \mathcal{M}}\|s-m\|$.

\section{Preliminaries on Topological Degree Theory}

Let $D \subset \mathbb{R}^{n}$ be an open and bounded set. Let $f \in C^{1}\left(D ; \mathbb{R}^{n}\right) \cap C^{0}\left(\bar{D} ; \mathbb{R}^{n}\right)$ be given. Set $A_{f}(D):=\{x \in \bar{D}: f(x)=0\}$ and $B_{f}(D):=\left\{x \in D: \operatorname{det} J_{f}(x)=0\right\}$ where $J_{f}(x)$ denotes the Jacobian Matrix of $f$ at $x$ defined by $\left(J_{f}\right)_{i j}:=\frac{\partial f_{i}}{\partial x_{j}}(x), i, j \in\{1, \ldots, n\}$. If $A_{f}(D) \cap B_{f}(D)=\emptyset$ and $0 \notin f(\partial D)$ then $A_{f}(D)$ is a finite set and the Brouwer topological degree of $f$ with respect to $D$ and 0 is well-defined by the formula

$$
\operatorname{deg}(f, D, 0)=\sum_{x \in A_{f}(D)} \operatorname{sgn}\left(\operatorname{det} J_{f}(x)\right)
$$

where $\operatorname{sgn}(t)=1$ for $t>0$ and $\operatorname{sgn}(t)=-1$ for $t<0$. More generally, if $f: \bar{D} \rightarrow \mathbb{R}^{n}$ is continuous and $0 \notin f(\partial D)$ then the Brouwer topological degree of $f$ with respect to $D$ and 0 is well-defined (see e.g. [18]) and denoted by $\operatorname{deg}(f, D, 0)$.

Let us now recall some properties of the topological degree we will use later in this paper.

1. If $0 \notin f(\partial D)$ and $\operatorname{deg}(f, D, 0) \neq 0$ then there exists $x \in D$ such that $f(x)=0$.

2. Let $\varphi:[0,1] \times \bar{B}_{r} \rightarrow \mathbb{R}^{n} ;(\lambda, x) \mapsto \varphi(\lambda, x)$, be continuous such that, for each $\lambda \in[0,1]$, one has $0 \notin \varphi(\lambda, \partial D)$. Then the map $\lambda \mapsto \operatorname{deg}(\varphi(\lambda,), D, 0$.$) is constant on [0,1]$.

3. We have

$$
\operatorname{deg}\left(i d_{\mathbb{R}^{n}}, D, 0\right)=1
$$

4. If $0 \notin f(\partial D)$ and $\alpha>0$ then

$$
\operatorname{deg}(\alpha f, D, 0)=\operatorname{deg}(f, D, 0)
$$


and

$$
\operatorname{deg}(-\alpha f, D, 0)=(-1)^{n} \operatorname{deg}(f, D, 0)
$$

5. Let $D^{\prime} \subset D$ be an open set such that $A_{f}(D) \subset D^{\prime}$. Then

$$
\operatorname{deg}(f, D, 0)=\operatorname{deg}\left(f, D^{\prime}, 0\right) .
$$

6. Let $A \in \mathbb{R}^{n \times n}$ be a nonsingular matrix. Then $\operatorname{deg}(A ., D, 0)=\operatorname{sgn}(\operatorname{det} A)$.

7. Let $f:[-r,+r] \rightarrow \mathbb{R}$ be a continuous function. Suppose that $f(-r) \neq 0$ and $f(+r) \neq 0$. Then

$$
\begin{gathered}
\operatorname{deg}(f,]-r,+r[, 0)=0 \text { if } f(-r) f(+r)>0, \\
\operatorname{deg}(f,]-r,+r[, 0)=+1 \text { if } f(-r)<0 \text { and } f(+r)>0, \\
\operatorname{deg}(f,]-r,+r[, 0)=-1 \text { if } f(-r)>0 \text { and } f(+r)<0 .
\end{gathered}
$$

\section{Generalized Projection Operator and its Inverse}

Let $\varphi: \mathbb{R}^{n} \rightarrow \mathbb{R} \cup\{\infty\}$ be a proper, convex and lower semi-continuous mapping. We denote by $\operatorname{dom}\{\varphi\}$ the domain of $\varphi$, i.e.

$$
\operatorname{dom}\{\varphi\}:=\left\{x \in \mathbb{R}^{n}: \varphi(x)<+\infty\right\} .
$$

We denote by $\partial \varphi$ the convex subdifferential of $\varphi$. Recall that

$$
\partial \varphi(x)=\left\{w \in \mathbb{R}^{n}: \varphi(v)-\varphi(x) \geq\langle w, v-x\rangle, \forall v \in \mathbb{R}^{n}\right\} .
$$

We denote by $D(\partial \varphi)$ the domain of $\partial \varphi$ and by $R(\partial \varphi)$ the range of $\partial \varphi$, i.e.

$$
D(\partial \varphi):=\left\{x \in \mathbb{R}^{n}: \partial \varphi(x) \neq \emptyset\right\}, \quad R(\partial \varphi):=\cup_{x \in \mathbb{R}^{n}} \partial \varphi(x) .
$$

Recall that

$$
D(\partial \varphi) \subset \operatorname{dom}\{\varphi\} \subset \overline{D(\partial \varphi)} .
$$

Let $y \in \mathbb{R}^{n}$ be given. We consider the variational inequality problem: Find $x \in \mathbb{R}^{n}$ such that

$$
\langle x-y, v-x\rangle+\varphi(v)-\varphi(x) \geq 0, \forall v \in \mathbb{R}^{n} .
$$

Problem (7) has a unique solution (see e.g. $[13,30]$ ) that we denote by $P_{\varphi}(y)$. The operator

$$
P_{\varphi}: \mathbb{R}^{n} \rightarrow \mathbb{R}^{n} ; y \mapsto P_{\varphi}(y)
$$

INRIA 
is thus well-defined. It is clear that

$$
P_{\varphi}\left(\mathbb{R}^{n}\right) \subset D(\partial \varphi)
$$

and

$$
P_{\varphi}(y)=\left(i d_{\mathbf{R}^{n}}+\partial \varphi\right)^{-1}(y) .
$$

If $\varphi \equiv \psi_{K}$ where $K$ is a nonempty closed convex set and $\psi_{K}$ denotes the indicator function of $K$, then

$$
P_{\varphi} \equiv P_{\psi_{K}} \equiv P_{K}
$$

where $P_{K}$ denotes the projection operator onto $K$ which is defined by the formula:

$$
\left\|x-P_{K} x\right\|=\min _{w \in K}\|x-w\| .
$$

Let $\Phi: \mathbb{R}^{n} \rightarrow \mathbb{R}^{n}$ be a continuous mapping and consider the inequality problem: Find $\bar{x} \in \mathbb{R}^{n}$ such that

$$
\langle\Phi(\bar{x}), v-\bar{x}\rangle+\varphi(v)-\varphi(\bar{x}) \geq 0, \forall v \in \mathbb{R}^{n} .
$$

It is clear that problem (9) is equivalent to the nonlinear equation: Find $\bar{x} \in \mathbb{R}^{n}$ such that

$$
\bar{x}-P_{\varphi}(\bar{x}-\Phi(\bar{x}))=0 .
$$

Proposition 1 The operator $P_{\varphi}$ is nonexpansive, i.e.

$$
\left\|P_{\varphi} v-P_{\varphi} y\right\| \leq\|v-y\|, \forall v, y \in \mathbb{R}^{n} .
$$

Proof: Let $v, y \in \mathbb{R}^{n}$ be given. We set $x:=P_{\varphi}(v)$ and $x^{*}:=P_{\varphi}(y)$. We have

$$
\langle x-v, w-x\rangle+\varphi(w)-\varphi(x) \geq 0, \forall w \in \mathbb{R}^{n}
$$

and

$$
\left\langle x^{*}-y, w-x^{*}\right\rangle+\varphi(w)-\varphi\left(x^{*}\right) \geq 0, \forall w \in \mathbb{R}^{n} .
$$

Setting $w:=x^{*}$ in (11) and $w:=x$ in (12), we obtain the relations $\left\langle x-v, x-x^{*}\right\rangle-$ $\varphi\left(x^{*}\right)+\varphi(x) \leq 0$ and $-\left\langle x^{*}-y, x-x^{*}\right\rangle-\varphi(x)+\varphi\left(x^{*}\right) \leq 0$, from wich we deduce that $\left\|x-x^{*}\right\|^{2} \leq\left\|x-x^{*}\right\|\|v-y\|$. It follows that

$$
\left\|P_{\varphi}(v)-P_{\varphi}(y)\right\| \leq\|v-y\| .
$$

RR $\mathrm{n}^{\circ} 5171$ 
Let us now denote by $\mathcal{A}_{\varphi}: \mathbb{R}^{n} \rightarrow 2^{\mathbb{R}^{n}}$ the set-valued operator defined by

$$
\mathcal{A}_{\varphi}(x)=\left\{f \in \mathbb{R}^{n}:\langle x-f, v-x\rangle+\varphi(v)-\varphi(x) \geq 0, \forall v \in \mathbb{R}^{n}\right\} .
$$

We see that

$$
\mathcal{A}_{\varphi}^{-1}(f)=P_{\varphi}(f)
$$

It is also easy to see that

$$
\left(t \mathcal{A}_{\varphi}\right)^{-1}(t f)=P_{\varphi}(f), \forall t>0
$$

Note that

$$
\mathcal{A}_{\varphi}(x)=\partial\left\{\frac{\|\cdot\|^{2}}{2}+\varphi(.)\right\}(x), \forall x \in \mathbb{R}^{n},
$$

so that $\mathcal{A}_{\varphi}$ is maximal monotone. It results that for any $t>0$, the operator $\left(i d_{\mathbf{R}^{n}}+t \mathcal{A}_{\varphi}\right)^{-1}$ is a well defined single-valued operator.

\section{Unilateral Dynamical Systems}

Let us first recall a general existence and uniqueness of solutions result (see e.g. [15]).

Theorem 1 . Let $\varphi: \mathbb{R}^{n} \rightarrow \mathbb{R} \cup\{+\infty\}$ be a proper, convex and lower semi-continuous function. Let $F: \mathbb{R}^{n} \rightarrow \mathbb{R}^{n}$ be a continuous operator such that for some $\bar{\omega} \in \mathbb{R}, F+\bar{\omega} I$ is monotone. Let $x_{0} \in \mathbb{R}^{n}$ be given. There exists a unique $u \in C^{0}\left([0,+\infty) ; \mathbb{R}^{n}\right)$ such that

$$
\begin{gathered}
\frac{d u}{d t} \in L_{\mathrm{loc}}^{\infty}\left(0,+\infty ; \mathbb{R}^{n}\right) ; \\
u \text { is right-differentiable on }[0,+\infty) ; \\
u(t) \in D(\partial \varphi), \forall t \geq 0 ; \\
\left\langle\frac{d u}{d t}(t)+F(u(t)), v-u(t)\right\rangle+\varphi(v)-\varphi(u(t)) \geq 0, \forall v \in \mathbb{R}^{n}, \text { a.e. } t \geq 0 ; \\
u(0)=x_{0} .
\end{gathered}
$$

INRIA 
Remark 1 Suppose that $F: \mathbb{R}^{n} \rightarrow \mathbb{R}^{n}$ can be written as

$$
F(x)=A x+\Phi^{\prime}(x)+F_{1}(x), \forall x \in \mathbb{R}^{n},
$$

where $A \in \mathbb{R}^{n \times n}$ is a real matrix, $\Phi \in C^{1}\left(\mathbb{R}^{n} ; \mathbb{R}\right)$ is convex and $F_{1}$ is Lipschitz continuous, i.e.

$$
\left\|F_{1}(x)-F_{1}(y)\right\| \leq k\|x-y\|, \forall x, y \in \mathbb{R}^{n},
$$

for some constant $k>0$. Then $F$ is continuous and $F+\bar{\omega} I$ is monotone provided that

$$
\bar{\omega} \geq \sup _{\|x\|=1}\langle-A x, x\rangle+k .
$$

The variational inequality in (19) can also be written as the differential inclusion

$$
\frac{d u}{d t}(t)+F(u(t)) \in-\partial \varphi(u(t)) \text {, a.e. } t \in[0, T],
$$

Suppose that the assumptions of Theorem 1 are satisfied and denote by $u\left(. ; x_{0}\right)$ the unique solution of Problem $P\left(x_{0}\right)$ (see (1)-(4)). Let us here also denote by $\mathcal{S}(F, \varphi)$ the set of stationary solutions of (19), i.e.

$$
\mathcal{S}(F, \varphi)=\left\{\bar{x} \in \mathbb{R}^{n}:\langle F(\bar{x}), w-\bar{x}\rangle+\varphi(w)-\varphi(\bar{x}) \geq 0, \forall w \in \mathbb{R}^{n}\right\} .
$$

Note that

$$
\mathcal{S}(F, \varphi)=\left\{\bar{x} \in \mathbb{R}^{n}: \bar{x}=P_{\varphi}(\bar{x}-F(\bar{x})\}\right.
$$

For $x_{0} \in D(\partial \varphi)$, we denote by $\gamma\left(x_{0}\right)$ the orbit

$$
\gamma\left(x_{0}\right):=\left\{u\left(\tau ; x_{0}\right) ; \tau \geq 0\right\} .
$$

Let us now assume without loss of generality that the trivial solution 0 is a stationary solution of (19), i.e.

$$
F(0) \in-\partial \varphi(0) .
$$

Then

$$
u(. ; 0) \equiv 0 .
$$

We may now define as in [15] the stability of the trivial solution. The stationary solution 0 is called stable if small perturbations of the initial condition $u\left(t_{0}\right)=0$ lead to solutions which remain in the neighborhood of 0 for all $t \geq 0$, precisely:

Definition 1 The equilibrium point $x=0$ is said to be stable in the sense of Lyapunov if for every $\varepsilon>0$ there exists $\eta=\eta(\varepsilon)>0$ such that for any $x_{0} \in D(\partial \varphi)$ with $\left\|x_{0}\right\| \leq \eta$ the solution $u\left(\cdot ; x_{0}\right)$ of problem $P\left(x_{0}\right)$ satisfies $\left\|u\left(t ; x_{0}\right)\right\| \leq \varepsilon, \forall t \geq 0$.

$\mathrm{RR} \mathrm{n}^{\circ} 5171$ 
If in addition the trajectories of the perturbed solutions are attracted by 0 then we say that the stationary solution is asymptotically stable, precisely:

Definition 2 The equilibrium point $x=0$ is asymptotically stable if (1) it is stable and (2) there exists $\delta>0$ such that for any $x_{0} \in D(\partial \varphi)$ with $\left\|x_{0}\right\| \leq \delta$ the solution $u\left(\cdot ; x_{0}\right)$ of problem $P\left(x_{0}\right)$ fulfills

$$
\lim _{t \rightarrow+\infty}\left\|u\left(t ; x_{0}\right)\right\|=0 .
$$

Definition 3 The equilibrium point $x=0$ is unstable if it is not stable.

The following result generalizes to unilateral systems the Lyapunov's direct method. The approach makes an essential use of some auxiliary function $V \in C^{1}\left(\mathbb{R}^{n} ; \mathbb{R}\right)$.

Theorem 2 ([15]) Suppose that the assumptions of Theorem 1 together with condition (22) hold. Suppose that there exists $\sigma>0$ and $V \in C^{1}\left(\mathbb{R}^{n} ; \mathbb{R}\right)$ such that

(1) $\quad V(0)=0$;

(2) $V(x)>0, \forall x \in \overline{D(\partial \varphi)} \cap \bar{B}_{\sigma}, x \neq 0$;

(3) $\left\langle F(x), V^{\prime}(x)\right\rangle+\varphi(x)-\varphi\left(x-V^{\prime}(x)\right) \geq 0, \forall x \in D(\partial \varphi) \cap \bar{B}_{\sigma}$.

Then the trivial solution of (16)-(19) is stable.

Remark 2 i) Assumptions (1) and (2) in Theorem 2 ensure the existence of a mapping $a:[0, \sigma] \rightarrow \mathbb{R}$ satisfying $a(0)=0$ and $a(t)>0, \forall t \in(0, \sigma]$ and such that $V(x) \geq a(\|x\|), \forall x \in$ $\overline{D(\partial \varphi)} \cap \bar{B}_{\sigma}$. The mapping a(.) defined by $a(r)=\inf \{V(x): r \leq\|x\| \leq \sigma\}(r \geq 0)$ is suitable.

ii) Assumption (3) in Theorem 2 implies that $\left\langle F(x), V^{\prime}(x)\right\rangle+\left\langle w, V^{\prime}(x)\right\rangle \geq 0, \forall w \in \partial \varphi(x), \forall x \in$ $D(\partial \varphi) \cap \bar{B}_{\sigma}$.

iii) If $K$ denotes a nonempty closed convex set and $\varphi \equiv \psi_{K}$ then assumption (3) in Theorem 2 implies that $\left\langle F(x), V^{\prime}(x)\right\rangle \geq 0, \forall x \in K \cap \bar{B}_{\sigma}$ and $x-V^{\prime}(x) \in K, \forall x \in K \cap \bar{B}_{\sigma}$. This last expression ensures that $-V^{\prime}(x) \in T_{K}(x), \forall x \in \partial K \cap \bar{B}_{\sigma}$ which characterizes the level sets of $V$ with respect to the boundary of $K$.

iv) A more general version of Theorem 2 is given in [15]. 
Let $V \in C^{1}\left(\mathbb{R}^{n} ; \mathbb{R}\right)$ be given. We set

$$
\mathcal{E}(F, \varphi, V):=\left\{x \in D(\partial \varphi):\left\langle F(x), V^{\prime}(x)\right\rangle+\varphi(x)-\varphi\left(x-V^{\prime}(x)\right)=0\right\} .
$$

Recall here that a set $\mathcal{D} \subset D(\partial \varphi)$ is invariant provided that

$$
x_{0} \in \mathcal{D} \Rightarrow \gamma\left(x_{0}\right) \subset \mathcal{D} .
$$

We recall a recent result that generalizes to unilateral systems the famous Krakovskii-LaSalle invariance principle.

Theorem 3 (Invariance Theorem [1]) Suppose that the assumptions of Theorem 1 hold. Let $\Psi \subset \mathbb{R}^{n}$ be a compact set and $V \in C^{1}\left(\mathbb{R}^{n} ; \mathbb{R}\right)$ a function such that

(1) $\varphi()-.\varphi\left(.-V^{\prime}().\right)$ is lower semicontinuous on $D(\partial \varphi) \cap \Psi$;

(2) $\left\langle F(x), V^{\prime}(x)\right\rangle+\varphi(x)-\varphi\left(x-V^{\prime}(x)\right) \geq 0, \forall x \in D(\partial \varphi) \cap \Psi$;

(3) $D(\partial \varphi)$ is closed.

We denote by $\mathcal{M}(F, \varphi, V, \Psi)$ the largest invariant subset of $\mathcal{E}(F, \varphi, V) \cap \Psi$. Then for each $x_{0} \in D(\partial \varphi)$ such that $\gamma\left(x_{0}\right) \subset \Psi$, we have

$$
\lim _{\tau \rightarrow+\infty} d\left(u\left(\tau ; x_{0}\right), \mathcal{M}(F, \varphi, V, \Psi)\right)=0
$$

\section{The Poincaré Operator}

Let $T>0$ be given. Theorem 1 enables us to define the one parameter family $\{S(t): 0 \leq$ $t \leq T\}$ of operators from $D(\partial \varphi)$ into $\mathbb{R}^{n}$, as follows:

$$
\forall y \in D(\partial \varphi), \quad S(t) y=x(t ; y),
$$

$x(. ; y)$ being the unique solution on $[0, T]$ of the evolution problem $P(y)$. Note that

$$
\forall y \in D(\partial \varphi), \quad S(0) y=y .
$$

According to (24), the unique solution of the problem (16 )-(19) satisfies, in addition, the periodicity condition

$$
u(0)=u(T)
$$

$\mathrm{RR} \mathrm{n}^{\circ} 5171$ 
if and only if $y$ is a fixed point of $S(T)$, that is

$$
S(T) y=y .
$$

Thus the problem of the existence of a periodic solution for the evolution problem (16)-(19) is reduced to that of the existence of a fixed point for $S(T)$. The operator $S(T)$ is called "Poincaré Operator".

Lemma 1 (see e.g. [30]) Let $0 \leq t_{1}<t_{2}<+\infty$ be given and let $a, b \in L^{1}\left(t_{1}, t_{2} ; \mathbb{R}\right.$ ) with $b(t) \geq 0$ a.e. $t \in\left[t_{1}, t_{2}\right]$. Let the absolutely continuous function $w:\left[t_{1}, t_{2}\right] \rightarrow \mathbb{R}_{+}$satisfy

$$
(1-\alpha) \frac{d w}{d t}(t) \leq a(t) w(t)+b(t) w^{\alpha}(t), \text { a.e. } t \in\left[t_{1}, t_{2}\right],
$$

where $0 \leq \alpha<1$. Then

$$
w^{1-\alpha}(t) \leq w^{1-\alpha}\left(t_{1}\right) e^{\int_{t_{1}}^{t} a(s) d s}+\int_{t_{1}}^{t} e^{\int_{s}^{t} a(q) d q} b(s) d s, \forall t \in\left[t_{1}, t_{2}\right] .
$$

Theorem 4 Suppose that the assumptions of Theorem 1 hold. Then for $0 \leq t_{1}<t_{2}<+\infty$, $y, z \in D(\partial \varphi)$, we have

$$
\left\|S\left(t_{2}\right) y-S\left(t_{2}\right) z\right\| \leq \sqrt{e^{2 \bar{\omega}\left(t_{2}-t_{1}\right)}}\left\|S\left(t_{1}\right) y-S\left(t_{1}\right) z\right\| .
$$

Proof: Let $0 \leq t_{1}<t_{2}<+\infty, y, z \in D(\partial \varphi)$ be given. We know that

$$
\begin{gathered}
\left\langle\frac{d}{d t} S(t) y+F(S(t) y), v-S(t) y\right\rangle+ \\
+\varphi(v)-\varphi(S(t) y) \geq 0, \forall v \in \mathbb{R}^{n}, \text { a.e. } t \geq 0
\end{gathered}
$$

and

$$
\begin{gathered}
\left\langle\frac{d}{d t} S(t) z+F(S(t) z), h-S(t) z\right\rangle+ \\
+\varphi(h)-\varphi(S(t) z) \forall h \in \mathbb{R}^{n}, \text { a.e. } t \geq 0 .
\end{gathered}
$$

Setting $v=S(t) z$ in (25) and $h=S(t) y$ in (26), we obtain the relations:

$$
-\left\langle\frac{d}{d t} S(t) y+F(S(t) y), S(t) z-S(t) y\right\rangle-\varphi(S(t) z)+\varphi(S(t) y) \leq 0, \text { a.e. } t \geq 0
$$


and

$$
\left\langle\frac{d}{d t} S(t) z+F(S(t) z), S(t) z-S(t) y\right\rangle-\varphi(S(t) y)+\varphi(S(t) z) \leq 0, \text { a.e. } t \geq 0
$$

It results that

$$
\begin{gathered}
\left\langle\frac{d}{d t}(S(t) z-S(t) y), S(t) z-S(t) y\right\rangle \leq \\
\leq \bar{\omega}\|S(t) z-S(t) y\|^{2}-\langle[F+\bar{\omega} I](S(t) z)-[F+\bar{\omega} I](S(t) y), S(t) z-S(t) y\rangle \text { a.e. } t \geq 0 .
\end{gathered}
$$

Our hypothesis ensure that $F+\bar{\omega} I$ is monotone. It results that

$$
\frac{d}{d t}\|S(t) z-S(t) y\|^{2} \leq 2 \bar{\omega}\|S(t) z-S(t) y\|^{2} \text {, a.e. } t \geq 0 \text {. }
$$

Using Lemma 1 with $w():.=\|S(.) z-S(.) y\|^{2}, a():.=2 \bar{\omega}, b()=0,. \alpha=0$, we get

$$
\left\|S\left(t_{2}\right) z-S\left(t_{2}\right) y\right\|^{2} \leq\left\|S\left(t_{1}\right) z-S\left(t_{1}\right) y\right\|^{2} e^{2 \bar{\omega}\left(t_{2}-t_{1}\right)} .
$$

The conclusion follows.

A consequence of Theorem 4 is that

$$
\|S(t) y-S(t) z\| \leq \sqrt{e^{2 \bar{\omega}}}\|y-z\|, \forall y, z \in D(\partial \varphi), t \in[0, T]
$$

The Poincaré operator $S(T): \mathbb{R}^{n} \rightarrow \mathbb{R}^{n} ; y \rightarrow S(T) y$ is thus Lipschitz continuous on $D(\partial \varphi)$, i.e.

$$
\|S(T) y-S(T) z\| \leq \sqrt{e^{2 \bar{\omega}} T}\|y-z\|, \forall y, z \in D(\partial \varphi) .
$$

Remark 3 i) Note that if $F$ is continuous and monotone then (28) holds with $\bar{\omega}=0$. In this case, the Poincaré operator $S(T)$ is nonexpansive on $D(\partial \varphi)$.

ii) If $F$ is continuous and strongly monotone, i.e. there exists $\alpha>0$ such that

$$
\langle F(x)-F(y), x-y\rangle \geq \alpha\|x-y\|^{2}, \forall x, y \in \mathbb{R}^{n}
$$

then (28) holds with $\bar{\omega}=-\alpha<0$ and the Poincaré operator $S(T)$ is a contraction on $D(\partial \varphi)$.

RR $n^{\circ} 5171$ 


\section{Necessary Conditions of Asymptotic Stability}

We suppose that:

$\left(h_{1}\right) \varphi: \mathbb{R}^{n} \rightarrow \mathbb{R} \cup\{+\infty\}$ is convex and lower semi-continuous;

$\left(h_{2}\right) D(\partial \varphi)$ is closed;

$\left(h_{3}\right) 0 \in D(\partial \varphi)$

$\left(h_{4}\right)$ There exists a neighborhood $\mathcal{N}$ of 0 and a constant $C_{1}>0$ such that

$$
\left|\varphi\left(x_{1}\right)-\varphi\left(x_{2}\right)\right| \leq C_{1}\left\|x_{1}-x_{2}\right\|, \forall x_{1}, x_{2} \in \mathcal{N} \cap \operatorname{dom}\{\varphi\}
$$

Condition $\left(h_{2}\right)$ together with (6) ensure that $D(\partial \varphi)=\operatorname{dom}\{\varphi\}$. Thus $D(\partial \varphi)$ is also convex. The projection operator $P_{D(\partial \varphi)}$ onto $D(\partial \varphi)$ is well-defined. Recall that

$$
\begin{gathered}
P_{D(\partial \varphi)}^{2}=P_{D(\partial \varphi)}, \\
\left\|P_{D(\partial \varphi)} x-P_{D(\partial \varphi)} y\right\| \leq\|x-y\|, \forall x, y \in \mathbb{R}^{n},
\end{gathered}
$$

and

$$
\left\langle P_{D(\partial \varphi)} x-x, w-P_{D(\partial \varphi)} x\right\rangle \geq 0, \forall w \in D(\partial \varphi) .
$$

We suppose also that

$\left(h_{5}\right) F: \mathbb{R}^{n} \rightarrow \mathbb{R}^{n}$ is a continuous operator such that for some $\bar{\omega} \in \mathbb{R}, F+\bar{\omega} I$ is monotone;

$\left(h_{6}\right) F$ is locally Lipschitz at 0.

Finally, we suppose that

$\left(h_{7}\right) 0$ is an isolated stationary solution of (19), i.e.

$$
0=P_{\varphi}(F(0))
$$

and there is a neighborhood $\mathcal{V}$ of 0 such that

$$
x \neq P_{\varphi}(x-F(x)), \forall x \in \mathcal{V} \backslash\{0\} .
$$

Note that the relation in (29) can also be written as

$$
\langle F(0), w\rangle+\varphi(w)-\varphi(0) \geq 0, \forall w \in \mathbb{R}^{n} .
$$

Let us now define the continuous mapping $\Lambda: \mathbb{R}^{n} \rightarrow \mathbb{R}^{n}$ by

$$
\Lambda(x):=x-P_{\varphi}(x-F(x)) .
$$

INRIA 
If $\left(h_{7}\right)$ is satisfied then for $r>0$ small enough, $\operatorname{deg}\left(\Lambda(),. B_{r}, 0\right)$ is well defined and constant. We set

$$
\zeta(\Lambda, 0):=\operatorname{deg}\left(\Lambda(.), B_{r}, 0\right) \text { for } r>0 \text { small enough. }
$$

Remark 4 If $n=1$ then $\zeta(\Lambda, 0)$ can be computed by using property 7 (see Section 2) of degree. More precisely,

$$
\begin{gathered}
\zeta(\Lambda, 0)=0 \text { if } \Lambda(-r) \Lambda(+r)>0, \text { for } r>0 \text { small enough, } \\
\zeta(\Lambda, 0)=+1 \text { if } \Lambda(-r)<0 \text { and } \Lambda(+r)>0 \text {, for } r>0 \text { small enough, } \\
\zeta(\Lambda, 0)=-1 \text { if } \Lambda(-r)>0 \text { and } \Lambda(+r)<0, \text { for } r>0 \text { small enough. }
\end{gathered}
$$

Example 1 i) Let $F$ and $\varphi$ be defined by $F(x)=2 x$ and $\varphi(x)=\Psi_{\mathbf{R}_{+}}$. Here

$$
\Lambda(x)=x-\max \{0,-x\} .
$$

For $r>0$, we have $\Lambda(-r)=-2 r<0$ and $\Lambda(r)=r>0$ and thus $\zeta(\Lambda, 0)=+1$.

ii) Let $F$ and $\varphi$ be defined by $F(x)=-2 x$ and $\varphi(x)=\Psi_{\mathbb{R}_{+}}$. Here

$$
\Lambda(x)=x-\max \{0,3 x\} .
$$

For $r>0$, we have $\Lambda(-r)=-r<0$ and $\Lambda(r)=-2 r<0$ and thus $\zeta(\Lambda, 0)=0$.

More generally, let us define for $\varepsilon>0$ the sets

$$
P_{i}(-\varepsilon):=\left\{x \in[-\varepsilon,+\varepsilon]^{n}: x_{i}=-\varepsilon\right\}, i \in\{1, \ldots, n\}
$$

and

$$
P_{i}(+\varepsilon):=\left\{x \in[-\varepsilon,+\varepsilon]^{n}: x_{i}=+\varepsilon\right\}, i \in\{1, \ldots, n\} .
$$

Note that

$$
\cup_{i=1}^{n}\left(P_{i}(-\varepsilon) \cup P_{i}(+\varepsilon)\right)=\partial(]-\varepsilon,+\varepsilon\left[^{n}\right)
$$

Proposition 2 Suppose that there exists $\varepsilon_{0}>0$ such that

$$
\Lambda_{i}(x) \Lambda_{i}(y)<0, \forall x \in P_{i}(-\varepsilon), y \in P_{i}(+\varepsilon), \epsilon \in\left(0, \varepsilon_{0}\right],(i \in\{1, \ldots, n\}) .
$$

Then

$$
\zeta(\Lambda, 0)=\prod_{i=1}^{n} \sigma_{i}
$$

where $\sigma_{i}:=\operatorname{sgn}\left(\Lambda_{i}\left(\bar{x}^{i}\right)\right)$ with $\bar{x}^{i}$ denoting any element of $P_{i}(+\bar{\epsilon})$ and $\bar{\epsilon}$ any real in $\left(0,+\varepsilon_{0}\right]$.

RR $n^{\circ} 5171$ 
Proof: Let $0<\varepsilon \leq \varepsilon_{0}$ be given. Assumption (33) together with the continuity of $\Lambda_{i}$ ensure that $\operatorname{sgn}\left(\Lambda_{i}().\right)$ is constant on $P_{i}(+\varepsilon)$. Let $x_{\varepsilon}^{i}$ be any element of $P_{i}(+\varepsilon)$, we get (see Theorem (c) in $[19])$ :

$$
\operatorname{deg}(\Lambda(.),]-\varepsilon,+\varepsilon\left[{ }^{n}, 0\right)=\prod_{i=1}^{n} \operatorname{sgn}\left(\Lambda_{i}\left(x_{\varepsilon}^{i}\right)\right) .
$$

Moreover condition (33) implies that $\Lambda(x) \neq 0, \forall x \in]-\varepsilon_{0},+\varepsilon_{0}\left[{ }^{n}, x \neq 0\right.$ and property 5 (see Section 2) of degree ensures that $\varepsilon \rightarrow \operatorname{deg}(\Lambda()]-,\varepsilon,+\varepsilon\left[{ }^{n}, 0\right)$ is constant on $\left(0,+\varepsilon_{0}\right]$.

Let $r_{0}>0$ be such that $\left.B_{r_{0}} \subset\right]-\varepsilon_{0},+\varepsilon_{0}\left[\right.$. Then $0 \notin \Lambda\left(\partial B_{r}\right)$ and $\zeta(\Lambda, 0)=\operatorname{deg}\left(\Lambda(),. B_{r}, 0\right)$ for all $0<r \leq r_{0}$. Let $r \in\left(0, r_{0}\right]$ be given, there exists $\varepsilon \equiv \varepsilon(r) \in(0, r)$ such that ]$-\varepsilon,+\varepsilon\left[{ }^{n} \subset B_{r}\right.$. It is also clear from (33) that $\left.A_{\Lambda}\left(B_{r}\right)=\{0\} \subset\right]-\varepsilon,+\varepsilon\left[{ }^{n}\right.$. Then using property 5 (see Section 2) of degree, we obtain

$$
\zeta(\Lambda, 0)=\operatorname{deg}(\Lambda(.),]-\varepsilon,+\varepsilon\left[^{n}, 0\right)=\operatorname{deg}(\Lambda(.),]-\bar{\varepsilon},+\bar{\varepsilon}\left[{ }^{n}, 0\right)=\prod_{i=1}^{n} \sigma_{i} .
$$

Proposition 3 Suppose that there exists $\varepsilon_{0}>0$ such that

$$
\Lambda_{i}(x) \Lambda_{i}(y)>0, \forall x \in P_{i}(-\varepsilon), y \in P_{i}(+\varepsilon), \epsilon \in\left(0, \varepsilon_{0}\right],(i \in\{1, \ldots, n\}) .
$$

Then

$$
\zeta(\Lambda, 0)=0
$$

Proof: Let $r_{0}>0$ such that $\left.B_{r} \subset\right]-\varepsilon_{0},+\varepsilon_{0}$ [. As in the proof of Proposition 2 we see that $\zeta(\Lambda, 0)=\operatorname{deg}\left(\Lambda(),. B_{r}, 0\right)$ for all $0<r \leq r_{0}$. Let $r \in\left(0, r_{0}\right)$ be given. There exists $\varepsilon \equiv \varepsilon(r) \in(0, r)$ such that

$$
\operatorname{deg}\left(\Lambda(.), B_{r}, 0\right)=\operatorname{deg}(\Lambda(.),]-\varepsilon,+\varepsilon\left[^{n}, 0\right) .
$$

Let us now consider the continuous homotopy $H:[0,1] \times[-\varepsilon,+\varepsilon]^{n} \rightarrow \mathbb{R}^{n}$ defined by

$$
H_{i}(\lambda, x)=\lambda\left(\Lambda_{i}\left(-\varepsilon e_{i}\right)+\frac{x_{i}+\varepsilon}{2 \varepsilon}\left(\Lambda_{i}\left(\varepsilon e_{i}\right)-\Lambda_{i}\left(-\varepsilon e_{i}\right)\right)\right)+(1-\lambda) \Lambda_{i}(x),
$$

where $e_{i}$ denotes the $i$-th unit vector of $\mathbb{R}^{n}$. We claim that if $x \in \partial(]-\varepsilon,+\varepsilon\left[{ }^{n}\right)$ and $\lambda \in[0,1]$ then $H(\lambda, x) \neq 0$. Indeed, if $x \in \partial(]-\varepsilon,+\varepsilon\left[^{n}\right)$ then either there exists $k \in\{1, \ldots, n\}$ such that $x \in P_{k}(-\varepsilon)$ or there exists $l \in\{1, \ldots, n\}$ such that $x \in P_{l}(+\varepsilon)$. If $x \in P_{k}(-\varepsilon)$ then $x_{k}=-\varepsilon$ and $H_{k}(\lambda, x)=\lambda \Lambda_{k}\left(-\varepsilon e_{k}\right)+(1-\lambda) \Lambda_{k}(x)$. Here $x$ and $-\varepsilon e_{k} \in P_{k}(-\varepsilon)$ and the continuity of $\Lambda$ together with (34) ensure that $\Lambda_{k}\left(-\varepsilon e_{k}\right)$ and $\Lambda_{k}(x)$ are nonzero and have the same sign. Thus $H_{k}(\lambda, x) \neq 0$ and therefore $H(\lambda, x) \neq 0$. If $x \in P_{l}(+\varepsilon)$ then $x_{l}=+\varepsilon$

INRIA 
and $H_{l}(\lambda, x)=\lambda \Lambda_{l}\left(\varepsilon e_{l}\right)+(1-\lambda) \Lambda_{l}(x)$. Here $x$ and $\varepsilon e_{l} \in P_{l}(-\varepsilon)$ and the continuity of $\Lambda$ together with (34) ensure that $\Lambda_{l}\left(\varepsilon e_{l}\right)$ and $\Lambda_{l}(x)$ are nonzero and have the same sign. It results that $H_{l}(\lambda, x) \neq 0$ and consequently $H(\lambda, x) \neq 0$. Thus from property 2 in Section 2 , we obtain

$$
\begin{gathered}
\zeta(\Lambda, 0)=\operatorname{deg}(\Lambda(.),]-\varepsilon,+\varepsilon\left[{ }^{n}, 0\right)= \\
=\operatorname{deg}(H(0, .),]-\varepsilon,+\varepsilon\left[^{n}, 0\right)=\operatorname{deg}(H(1, .),]-\varepsilon,+\varepsilon\left[{ }^{n}, 0\right) .
\end{gathered}
$$

It is now clear that $H(1,) \neq$.0 on $]-\varepsilon,+\varepsilon\left[{ }^{n}\right.$. Indeed,

$$
\begin{gathered}
H_{i}(1, x)=\Lambda_{i}\left(-\varepsilon e_{i}\right)+\frac{x_{i}+\varepsilon}{2 \varepsilon}\left(\Lambda_{i}\left(\varepsilon e_{i}\right)-\Lambda_{i}\left(-\varepsilon e_{i}\right)\right)= \\
=\left(1-\frac{x_{i}+\varepsilon}{2 \varepsilon}\right) \Lambda_{i}\left(-\varepsilon e_{i}\right)+\frac{x_{i}+\varepsilon}{2 \varepsilon} \Lambda_{i}\left(\varepsilon e_{i}\right),
\end{gathered}
$$

and $\frac{x_{i}+\varepsilon}{2 \varepsilon} \in(0,1)$ for $\left.x \in\right]-\varepsilon,+\varepsilon\left[{ }^{n}\right.$. Thus $H_{i}(1, x) \neq 0$ since condition (34) entails that $\Lambda_{i}\left(-\varepsilon e_{i}\right)$ and $\Lambda_{i}\left(\varepsilon e_{i}\right)$ are nonzero and have same sign. It results from property 1 in Section 2 , that $\operatorname{deg}(H(1,)]-,\varepsilon,+\varepsilon\left[^{n}, 0\right)=0$. The conclusion follows.

Example 2 i) Let $F$ and $\varphi$ be defined by $F\left(x_{1}, x_{2}\right)=\left(2 x_{1}, 3 x_{2}\right)$ and $\varphi(x)=\Psi_{\mathbf{R}_{+} \times \mathbb{R}_{+}}$. Here

$$
\Lambda(x)=\left(x_{1}-\max \left\{0,-x_{1}\right\}, x_{2}-\max \left\{0,-2 x_{2}\right\}\right) \text {. }
$$

For $\varepsilon>0$, we see that $\Lambda_{1}(x)=-2 \varepsilon<0$ if $x \in P_{1}(-\varepsilon), \Lambda_{1}(x)=\varepsilon>0$ if $x \in P_{1}(+\varepsilon)$, $\Lambda_{2}(x)=-3 \varepsilon<0$ if $x \in P_{2}(-\varepsilon)$ and $\Lambda_{2}(x)=\varepsilon>0$ if $x \in P_{2}(+\varepsilon)$. Applying Proposition 2 , we get $\zeta(\Lambda, 0)=1$.

ii) Let $F$ and $\varphi$ be defined by $F\left(x_{1}, x_{2}\right)=\left(-2 x_{1}+x_{2},-x_{2}\right)$ and $\varphi(x)=\Psi_{\mathbb{R}_{+} \times \mathbb{R}_{+}}$. Here

$$
\Lambda(x)=\left(x_{1}-\max \left\{0,3 x_{1}-x_{2}\right\}, x_{2}-\max \left\{0,2 x_{2}\right\}\right) .
$$

For $\varepsilon>0$, we see that $\Lambda_{1}(x)=-\varepsilon<0$ if $x \in P_{1}(-\varepsilon), \Lambda_{1}(x)=x_{2}-2 \varepsilon \leq-\varepsilon<0$ if $x \in P_{1}(+\varepsilon), \Lambda_{2}(x)=-\varepsilon<0$ if $x \in P_{2}(-\varepsilon)$ and $\Lambda_{2}(x)=-\varepsilon<0$ if $x \in P_{2}(+\varepsilon)$. Applying Proposition 3, we obtain $\zeta(\Lambda, 0)=0$.

The use of Propositions 2 and 3 is of particular interest if $\varphi \equiv \Psi_{\mathbb{R}_{+}^{n}}$ since in this case $\Lambda_{i}$ can be easily evaluated by the formula

$$
\Lambda_{i}(x)=x_{i}-\max \left\{0, x_{i}-F_{i}(x)\right\}=\min \left\{x_{i}, F_{i}(x)\right\} .
$$

Let us now prove our main result by following a methodology that has been originated by Quittner [25] in the framework of parabolic variational inequalities involving unilateral constraints defined by a closed and convex set. 
Theorem 5 Suppose that assumptions $\left(h_{1}\right)-\left(h_{7}\right)$ are satisfied. If the trivial stationary solution of (19) is asymptotically stable then

$$
\zeta(\Lambda, 0)=1
$$

Proof: The mapping $F($.$) is locally Lipschitz at 0$ and thus there exists $\delta_{0}>0$ and $C_{0}>0$ such that

$$
\|F(x)-F(y)\| \leq C_{0}\|x-y\|, \forall x, y \in B_{\delta_{0}} .
$$

$>$ From assumption $\left(h_{4}\right)$, we see that there exists $\delta_{1}>0$ and $C_{1}>0$ such that

$$
|\varphi(x)-\varphi(y)| \leq C_{1}\|x-y\|, \forall x, y \in \operatorname{dom}\{\varphi\} \cap B_{\delta_{1}} .
$$

The trivial stationary solution is isolated and thus, there exists $\delta_{2}>0$ such that

$$
x \neq P_{\varphi}(x-F(x)), \forall x \in B_{\delta_{2}} \backslash\{0\} .
$$

The trivial stationary solution is stable and thus, there exists $\delta_{3}>0$ such that

$$
\left|S(t) x_{0}\right| \leq \min \left\{1, \delta_{0}, \frac{\delta_{1}}{2\left(C_{0}+1\right)}\right\}, \forall x_{0} \in B_{\delta_{3}} \cap D(\partial \varphi) .
$$

The trivial stationary solution is attractive and thus, there exists $\delta_{4}>0$ such that

$$
\lim _{t \rightarrow+\infty} S(t) x_{0}=0, \forall x_{0} \in B_{\delta_{4}} \cap D(\partial \varphi) .
$$

Set

$$
r_{0}:=\min \left\{\frac{\delta_{0}}{2}, \frac{\delta_{1}}{2\left(1+C_{0}\right)}, \delta_{2}, \delta_{3}, \delta_{4}\right\}
$$

Let $0<r \leq r_{0}$ be given. From (38), we get the existence of $\eta:=\eta(r)>0$ such that

$$
\left\|x-P_{\varphi}(x-F(x))\right\| \geq \eta, \quad \frac{r}{8} \leq\|x\| \leq r .
$$

We set

$$
G:=i d_{\mathbb{R}^{n}}-F,
$$

so that $\Lambda=i d_{\mathbb{R}^{n}}-P_{\varphi}(G)$.

To prove our result, we will use the following claims. The proofs of these claims are given in the appendix.

CLAIM 1.

$$
\operatorname{deg}\left(i d_{\mathbb{R}^{n}}-S(t) P_{D(\partial \varphi)}, B_{r}, 0\right)=1, \forall t>0
$$


where $S($.$) is defined in Section 5$.

CLAIM 2.

$$
\begin{gathered}
\operatorname{deg}\left(i d_{\mathbb{R}^{n}}-P_{\varphi} G\left(P_{D(\partial \varphi)} \cdot\right), B_{r}, 0\right)= \\
=\operatorname{deg}\left(i d_{\mathbb{R}^{n}}-\left(i d_{\mathbb{R}^{n}}+t \mathcal{A}_{\varphi}\right)^{-1}\left(i d_{\mathbb{R}^{n}}+t G\right) P_{D(\partial \varphi)} \cdot, B_{r}, 0\right), \forall t>0 .
\end{gathered}
$$

CLAIM 3.

$$
\operatorname{deg}\left(i d_{\mathbf{R}^{n}}-P_{\varphi} G\left(P_{D(\partial \varphi)} .\right), B_{r}, 0\right)=\operatorname{deg}\left(i d_{\mathbf{R}^{n}}-P_{\varphi} G(.), B_{r}, 0\right) .
$$

CLAIM 4. Let $x \in \partial B_{r}\left(r \leq r_{0}\right)$ be given. We set

$$
u(t):=S(t) P_{D(\partial \varphi)} x, t \geq 0,
$$

and

$$
v(t):=\left(i d_{\mathbb{R}^{n}}+t \mathcal{A}_{\varphi}\right)^{-1}\left(i d_{\mathbb{R}^{n}}+t G\right) P_{D(\partial \varphi)} x, t>0 .
$$

The following estimations hold:

(a) $\left\|P_{D(\partial \varphi)} x\right\| \leq r_{0}$;

(b) $\left\|P_{\varphi} G\left(P_{D(\partial \varphi)} x\right)\right\| \leq \delta_{1}$;

(c) $\|u(t)\| \leq \min \left\{1, \delta_{0}, \frac{\delta_{1}}{2\left(C_{0}+1\right)}\right\}, \forall t \geq 0$;

(d) $\left\|u(t)-P_{D(\partial \varphi)} x\right\| \leq \theta \sqrt{t}, \forall t \geq 0$, with

$$
\theta:=\sqrt{2\left(r_{0}+1\right)\left(C_{1}+\bar{\omega}\left(r_{0}+1\right)+C_{0} r_{0}+\|F(0)\|\right)} ;
$$

(e) $\left\|P_{\varphi} G(u(t))\right\| \leq \delta_{1}, \forall t \geq 0$;

(f) Let $t^{*} \in\left(0, \frac{1}{2 \bar{\omega}} \ln (4)\right]$ be given. If $\left\|u\left(t^{*}\right)\right\| \geq \frac{r}{4}$ then $\|u(t)\| \geq \frac{r}{8}, \forall t \in\left(0, t^{*}\right]$.

(g) $v(t) \in D(\partial \varphi)$ and

$$
\begin{gathered}
\left\langle\frac{v(t)-P_{D(\partial \varphi)} x}{t}, w-v(t)\right\rangle+\left\langle v(t)-G\left(P_{D(\partial \varphi)} x\right), w-v(t)\right\rangle+ \\
+\varphi(w)-\varphi(v(t)) \geq 0, \forall w \in \mathbb{R}^{n}, \forall t>0
\end{gathered}
$$

(h) $\left\|v(t)-P_{D(\partial \varphi)} x\right\| \leq \bar{\theta} \sqrt{t} ; \forall t>0$, with

$$
\bar{\theta}:=\sqrt{\frac{\left(C_{0} r_{0}\right)^{2}}{2}+\|F(0)\| r_{0}+C_{1} r_{0}} .
$$

$\mathrm{RR} \mathrm{n}^{\circ} 5171$ 
Set

$$
T^{*}:=\min \left\{\left(\frac{\delta_{0}}{2 \bar{\theta}}\right)^{2},\left(\frac{\delta_{1}}{2\left(1+C_{0}\right) \bar{\theta}}\right)^{2}\right\}
$$

Then

(i) $\|v(t)\| \leq \min \left\{\delta_{0}, \frac{\delta_{1}}{1+C_{0}}\right\}, \forall t \in\left(0, T^{*}\right]$;

(j) $\| P_{\varphi}\left(G(v(t)) \| \leq \delta_{1}, \forall t \in\left(0, T^{*}\right]\right.$.

$C L A I M$ 5. Let $0<r \leq r_{0}$ be given and set

$$
C:=\max \left\{\frac{2}{3} \theta\left(1+C_{0}\right)\left(\|F(0)\|+C_{0}+C_{1}\right), \bar{\theta}\left(1+C_{0}\right)\left(\|F(0)\|+\delta_{1}+\left(1+C_{0}\right) \delta_{0}+C_{1}\right)\right\}
$$

and

$$
\bar{T}:=\min \left\{\frac{1}{2 \bar{\omega}} \ln (4), T^{*}, \frac{\eta^{4}}{2 C^{2}}, \frac{\eta^{4}}{2 C^{2}\left(\nu^{*}+1\right)^{2}}, \frac{\eta^{4}}{2 C^{2}\left(\frac{1}{\nu_{*}}+1\right)^{2}}\right\},
$$

with $\nu^{*}=\frac{4}{3} \frac{\left(\delta_{0}+r\right)}{r}, \nu_{*}=\frac{7 r}{8(1+r)}, \bar{\omega}$ in Theorems 1 and $4, T^{*}$ in Claim $4(\mathrm{~h})$ and $\eta$ in (41). For any $t \in(0, \bar{T}]$, we have

$$
\begin{gathered}
\operatorname{deg}\left(i d_{\mathbb{R}^{n}}-\left(i d_{\mathbb{R}^{n}}+t \mathcal{A}_{\varphi}\right)^{-1}\left(i d_{\mathbb{R}^{n}}+t G\right) P_{D(\partial \varphi)} ., B_{r}, 0\right)= \\
=\operatorname{deg}\left(i d_{\mathbb{R}^{n}}-S(t) P_{D(\partial \varphi) \cdot}, B_{r}, 0\right) .
\end{gathered}
$$

We are now able to use Claims 1-5 in order to conclude the proof of our result. Let $0<r \leq r_{0}$ be given and compute $\bar{T} \equiv \bar{T}(r)$ as in Claim 5 . We have

$$
\zeta(\Lambda, 0)=\operatorname{deg}\left(\Lambda, B_{r}, 0\right)=\operatorname{deg}\left(i d_{\mathbb{R}^{n}}-P_{\varphi} G(.), B_{r}, 0\right) .
$$

$>$ From Claim 3 and (43), we see that

$$
\zeta(\Lambda, 0)=\operatorname{deg}\left(i d_{\mathbb{R}^{n}}-P_{\varphi} G\left(P_{D(\partial \varphi)} .\right), B_{r}, 0\right) .
$$

Let us now choose $t \in(0, \bar{T}]$. From Claim 2 and (44), we obtain

$$
\zeta(\Lambda, 0)=\operatorname{deg}\left(i d_{\mathbb{R}^{n}}-\left(i d_{\mathbb{R}^{n}}+t \mathcal{A}_{\varphi}\right)^{-1}\left(i d_{\mathbb{R}^{n}}+t G\right) P_{D(\partial \varphi)}, B_{r}, 0\right) .
$$

Claim 5 and (45) ensure that

$$
\zeta(\Lambda, 0)=\operatorname{deg}\left(i d_{\mathbb{R}^{n}}-S(t) P_{D(\partial \varphi)} ., B_{r}, 0\right) .
$$

Finally, Claim 1 and (46) yield

$$
\zeta(\Lambda, 0)=1
$$

The following result is an equivalent formulation of Theorem 5 .

INRIA 
Corollary 1 Suppose that assumptions $\left(h_{1}\right)-\left(h_{7}\right)$ are satisfied. If $\zeta(\Lambda, 0) \neq 1$ then the trivial stationary solution of (19) is not asymptotically stable.

Example 3 i) The necessary condition of asymptotic stability $\zeta(\Lambda, 0)=1$ is satisfied with the data given in Example 1 (i) and Example 2 (i).

ii) The trivial stationary solution of (19) with the data given in Example 1 (ii) or Example 2 (ii) is not asymptotically stable.

\section{The case $\varphi=\psi_{K}$ whith $K$ closed and convex}

It is known that if $K$ is a polyhedron, then the system in (19) is equivalent to a complementarity system [12], whose study has a significant interest for control applications. Let us first state a result of Quittner [25].

Lemma 2 (Quittner) Let $K \subset \mathbb{R}^{n}$ be a closed and convex set such that $0 \in K$. Set

$$
K_{0}:=\overline{\cup_{\alpha>0} \alpha K}
$$

Suppose that $G: \mathbb{R}^{n} \rightarrow \mathbb{R}^{n}$ satisfies

(1) $G$ is continuous;

(2) $\quad G(0)=0$;

(3) $J_{G}(0)$ exists;

(4) $\operatorname{deg}\left(i d_{\mathbf{R}^{n}}-P_{K_{0}}\left(J_{G}(0).\right), B_{r}, 0\right)$ exists for $r>0$ small enough.

Then, for all $r>0$ sufficiently small, $0 \notin\left(i d_{\mathbb{R}^{n}}-P_{K}(G()).\right)\left(\partial B_{r}\right)$ and

$$
\operatorname{deg}\left(i d_{\mathbb{R}^{n}}-P_{K}(G(.)), B_{r}, 0\right)=\operatorname{deg}\left(i d_{\mathbf{R}^{n}}-P_{K_{0}}\left(J_{G}(0) .\right), B_{r}, 0\right) .
$$

Note that the original result of Quittner has been stated in a more general framework for compact continuous mapping $G$ defined on Hilbert spaces.

Let us now remark that Lemma 2 can be used together with Corollary 1 and Propositions 2 and 3.

$\mathrm{RR} \mathrm{n}^{\circ} 5171$ 
Example 4 Let $F$ and $K$ be defined by $F\left(x_{1}, x_{2}\right)=\left(-2 \sin \left(x_{1}\right)+x_{2},-\sin \left(x_{2}\right)\right)$ and $K=$ $\left\{\left(x_{1}, x_{2}\right) \in \mathbb{R}^{2}: 0 \leq x_{2} \leq x_{1}\right\}$. Then

$$
K_{0}=\mathbb{R}_{+} \times \mathbb{R}_{+}
$$

and

$$
J_{F}(0)=\left(\begin{array}{rr}
-2 & 1 \\
0 & -1
\end{array}\right) .
$$

Using the results of Example 2(ii) and Lemma 2, we get $\zeta(\Lambda, 0)=0$. It results that the trivial stationary solution of (19) is not asymptotically stable.

Let us here remark that if $K \subset \mathbb{R}^{n}$ is a closed convex set with $0 \in K$ then $\varphi:=\psi_{K}$ satisfies conditions $\left(h_{1}\right)-\left(h_{4}\right)$ of Section 6 . We see also that if the assumptions of Lemma 2 are satisfied for $G:=i d_{\mathbf{R}^{n}}-F$ then condition $\left(h_{7}\right)$ is also satisfied.

Theorem 6 Let $K \subset \mathbb{R}^{n}$ be a closed and convex set such that $0 \in K$ and $K_{0} \backslash\{0\} \neq \emptyset$ with $K_{0}$ in (48). Suppose that conditions $\left(h_{5}\right)-\left(h_{6}\right)$ are satisfied. Suppose also that $F(0)=0$ and $J_{F}(0)$ exists.

If there exists $\mu<0$ and $U_{\mu} \in K_{0} \backslash\{0\}$ such that

(1) $\left\langle U_{\mu}, h\right\rangle>0, \forall h \in K_{0} \backslash\{0\}$;

(2) $J_{F}(0)^{T} U_{\mu}=\mu U_{\mu}$.

Then the trivial stationary solution of (19) is not asymptotically stable.

Proof: Set $A:=I-J_{F}(0)$ and define $h:[0,1] \times \mathbb{R}^{n} \rightarrow \mathbb{R}^{n}$ by $h(\lambda, x)=\lambda x-P_{K_{0}}(\lambda A x+$ $\left.(1-\lambda) U_{\mu}\right)$. We claim that if $x \in \partial B_{r}, r>0$ and $\lambda \in[0,1]$ then $h(\lambda, x) \neq 0$. Indeed, suppose on the contrary that

$$
\lambda x=P_{K_{0}}\left(\lambda A x+(1-\lambda) U_{\mu}\right) .
$$

Then $\lambda \neq 0$ since otherwise get $0=P_{K_{0}}\left(U_{\mu}\right)=U_{\mu}$ and a contradiction. Thus $\lambda \neq 0$ and since $K_{0}$ is a cone, we get

$$
x=\frac{1}{\lambda} P_{K_{0}}\left(\lambda A x+(1-\lambda) U_{\mu}\right)=P_{K_{0}}\left(A x+\frac{(1-\lambda)}{\lambda} U_{\mu}\right) .
$$

Thus $x \in K_{0}$ and

$$
\left\langle x-A x-\frac{(1-\lambda)}{\lambda} U_{\mu}, v-x\right\rangle \geq 0, \forall v \in K_{0} .
$$

Setting $v:=x+U_{\mu} \in K_{0}+K_{0} \subset K_{0}$, we obtain

$$
\left\langle J_{F}(0) x, U_{\mu}\right\rangle \geq \frac{(1-\lambda)}{\lambda}\left\|U_{\mu}\right\|^{2} \geq 0 .
$$

INRIA 
Thus

$$
\left\langle x, J_{F}(0)^{T} U_{\mu}\right\rangle=\mu\left\langle x, U_{\mu}\right\rangle \geq 0 .
$$

Here since $x \in \partial B_{r}, r>0$, then $x \in K_{0} \backslash\{0\}$ and thus from assumption (1), we obtain that $\left\langle x, U_{\mu}\right\rangle>0$ so that $\mu \geq 0$ and a contradiction.

Then

$$
\begin{gathered}
\operatorname{deg}\left(i d_{\mathbb{R}^{n}}-P_{K_{0}}\left(i d_{\mathbb{R}^{n}}-J_{F}(0) .\right), B_{r}, 0\right)=\operatorname{deg}\left(h(1, .), B_{r}, 0\right)= \\
=\operatorname{deg}\left(h(0, .), B_{r}, 0\right)=\operatorname{deg}\left(-P_{K_{0}}\left(U_{\mu}\right), B_{r}, 0\right)=\operatorname{deg}\left(-U_{\mu}, B_{r}, 0\right)=0 .
\end{gathered}
$$

Using Lemma 2, we see that $\zeta(\Lambda, 0)=0$ and the conclusion follows from Corollary 1.

Example 5 Let $F$ and $K$ be defined by $F\left(x_{1}, x_{2}\right)=\left(-2 \sin \left(x_{1}\right)-10 x_{2}, \cos \left(x_{1}\right)-\sin \left(x_{2}\right)-1\right)$ and $K=\left\{\left(x_{1}, x_{2}\right) \in \mathbb{R}^{2}: 0 \leq x_{2} \leq x_{1}\right\}$. Then

$$
K_{0}=\mathbb{R}_{+} \times \mathbb{R}_{+}
$$

and

$$
J_{F}(0)=\left(\begin{array}{rr}
-2 & -10 \\
0 & -1
\end{array}\right) .
$$

Theorem 6 can be applied to ensure that the trivial stationary solution of (19) is not asymptotically stable. It is indeed easy to see that $\mu=-2$ is an eigenvalue of $J_{F}(0)^{T}$ and that the corresponding eigenvector $U_{\mu}=(1,10)^{T}$ satisfies conditions (1) and (2) of Theorem 6 .

Theorem 7 Let $K \subset \mathbb{R}^{n}$ be a closed and convex set such that $0 \in K$ and $K_{0}^{*} \cap K_{0} \backslash\{0\} \neq \emptyset$. Suppose that conditions $\left(h_{5}\right)-\left(h_{6}\right)$ are satisfied. Suppose also that $F(0)=0, J_{F}(0)$ exists and

(1) $\left\langle J_{F}(0) x, x\right\rangle<0, \forall x \in K_{0}, x \neq 0$,

then the trivial stationary solution of (19) is not asymptotically stable.

Proof: Let $U \in K_{0}^{*} \cap K_{0} \backslash\{0\}$ be fixed. Set $A:=I-J_{F}(0)$ and define $h:[0,1] \times \mathbb{R}^{n} \rightarrow \mathbb{R}^{n}$ by $h(\lambda, x)=\lambda x-P_{K_{0}}(\lambda A x+(1-\lambda) U)$. We claim that if $x \in \partial B_{r}, r>0$ and $\lambda \in[0,1]$ then $h(\lambda, x) \neq 0$. Indeed, suppose on the contrary that $\lambda x=P_{K_{0}}(\lambda A x+(1-\lambda) U)$. It is clear that $\lambda \neq 0$. Indeed, suppose that $\lambda=0$. Then $0=P_{K_{0}} U=U$ and a contradiction. Thus $\lambda \neq 0$ and

$$
x=P_{K_{0}}\left(A x+\frac{(1-\lambda)}{\lambda} U\right) .
$$

Thus $x \in K_{0}$ and

$$
\left\langle x-A x-\frac{(1-\lambda)}{\lambda} U, v-x\right\rangle \geq 0, \forall v \in K_{0} .
$$

$\mathrm{RR} \mathrm{n}^{\circ} 5171$ 
Setting $v:=2 x$, we obtain since $U \in K_{0}^{*}$

$$
\left\langle J_{F}(0) x, x\right\rangle \geq \frac{(1-\lambda)}{\lambda}\langle U, x\rangle \geq 0 .
$$

Here $x \in K_{0} \backslash\{0\}$ and from assumption (1), we obtain $\left\langle J_{F}(0) x, x\right\rangle<0$ which is a contradiction to (49).

Then

$$
\begin{aligned}
& \operatorname{deg}\left(i d_{\mathbb{R}^{n}}-P_{K_{0}}\left(i d_{\mathbb{R}^{n}}-J_{F}(0) .\right), B_{r}, 0\right)=\operatorname{deg}\left(h(1, .), B_{r}, 0\right)= \\
= & \operatorname{deg}\left(h(0, .), B_{r}, 0\right)=\operatorname{deg}\left(-P_{K_{0}}(U), B_{r}, 0\right)=\operatorname{deg}\left(-U, B_{r}, 0\right)=0 .
\end{aligned}
$$

Using Lemma 2, we see that $\zeta(\Lambda, 0)=0$ and the conclusion follows from Corollary 1 .

Remark 5 It is clear that if the matrix $J_{F}(0)$ is negative definite, then assumption (1) in Theorem 7 is satisfied.

Example 6 Let $F$ and $K$ be defined as in Example 4. Theorem 7 can also be applied to ensure that the trivial stationary solution of (19) is not asymptotically stable. Indeed, here $K_{0} \cap K_{0}^{*} \backslash\{0\}=\mathbb{R}_{+} \times \mathbb{R}_{+} \backslash\{(0,0)\}$ and the matrix $J_{F}(0)$ is negative definite.

\section{The case $\varphi$ convex and $\operatorname{dom}\{\varphi\}=\mathbb{R}^{n}$}

Let us here suppose that $\varphi: \mathbb{R}^{n} \rightarrow \mathbb{R}$ is convex with $\operatorname{dom}\{\varphi\}=\mathbb{R}^{n}$. Then $\varphi$ is continuous and $D(\partial \varphi)=\mathbb{R}^{n}$ (see Theorem 10.1 in [26]). Moreover $\varphi$ is Lipschitzian, relative to every bounded set (see Theorem 10.4 in [26]). It results that $\varphi$ satisfies conditions $\left(h_{1}\right)-\left(h_{4}\right)$ of Section 6 .

Proposition 4 Let $L>0$ be given and assume that $G:[0, L] \times \mathbb{R}^{n} \rightarrow \mathbb{R}^{n} ;(\lambda, y) \mapsto G(\lambda, y)$ is a continuous function on $[0, L] \times \mathbb{R}^{n}$. Then the application

$$
(\lambda, y) \mapsto P_{\lambda \varphi}(G(\lambda, y))
$$

is continuous on $[0, L] \times \mathbb{R}^{n}$.

Proof: Let $\left\{y_{n}\right\} \subset \mathbb{R}^{n}$ and $\left\{\lambda_{n}\right\} \subset[0, L]$ be given sequences such that $y_{n} \rightarrow y^{*}$ and $\lambda_{n} \rightarrow \lambda^{*}$ as $n \rightarrow+\infty$. We claim that $P_{\lambda_{n} \varphi}\left(G\left(\lambda_{n}, y_{n}\right)\right) \rightarrow P_{\lambda^{*} \varphi}\left(G\left(\lambda^{*}, y^{*}\right)\right)$ as $n \rightarrow+\infty$. Indeed, setting $x_{n}:=P_{\lambda_{n} \varphi}\left(G\left(\lambda_{n}, y_{n}\right)\right)$ and $x^{*}:=P_{\lambda^{*} \varphi}\left(G\left(\lambda^{*}, y^{*}\right)\right)$, we have

$$
\left\langle x_{n}-G\left(\lambda_{n}, y_{n}\right), v-x_{n}\right\rangle+\lambda_{n} \varphi(v)-\lambda_{n} \varphi\left(x_{n}\right) \geq 0, \forall v \in \mathbb{R}^{n}
$$


and

$$
\left\langle x^{*}-G\left(\lambda^{*}, y^{*}\right), v-x^{*}\right\rangle+\lambda^{*} \varphi(v)-\lambda^{*} \varphi\left(x^{*}\right) \geq 0, \forall v \in \mathbb{R}^{n} .
$$

Let us first check that the sequence $\left\{x_{n}\right\}$ is bounded. Indeed, suppose on the contrary that $\left\|x_{n}\right\| \rightarrow+\infty$ as $n \rightarrow+\infty$. Setting $v:=0$ in (50), we obtain

$$
-\left\langle x_{n}-G\left(\lambda_{n}, y_{n}\right), x_{n}\right\rangle+\lambda_{n}\left[\varphi(0)-\varphi\left(x_{n}\right)\right] \geq 0 .
$$

and thus

$$
\left\|x_{n}\right\|^{2} \leq\left\|G\left(\lambda_{n}, y_{n}\right)\right\|\left\|x_{n}\right\|+\lambda_{n}\left[\varphi(0)-\varphi\left(x_{n}\right)\right]
$$

It results that

$$
1 \leq \frac{\left\|G\left(\lambda_{n}, y_{n}\right)\right\|}{\left\|x_{n}\right\|}+\frac{\lambda_{n}}{\left\|x_{n}\right\|^{2}}\left[\varphi(0)-\varphi\left(x_{n}\right)\right] .
$$

For $n$ large enough, $\frac{1}{\left\|x_{n}\right\|} \in(0,1]$ and using the convexity of $\varphi$, we get

$$
\varphi\left(\frac{x_{n}}{\left\|x_{n}\right\|}\right) \leq \frac{1}{\left\|x_{n}\right\|} \varphi\left(x_{n}\right)+\left(1-\frac{1}{\left\|x_{n}\right\|}\right) \varphi(0) .
$$

and thus

$$
\frac{\varphi(0)-\varphi\left(x_{n}\right)}{\left\|x_{n}\right\|} \leq \varphi(0)-\varphi\left(\frac{x_{n}}{\left\|x_{n}\right\|}\right) .
$$

We obtain

$$
1 \leq \frac{\left\|G\left(\lambda_{n}, y_{n}\right)\right\|}{\left\|x_{n}\right\|}+\lambda_{n}\left[\frac{\varphi(0)-\varphi\left(\frac{x_{n}}{\left\|x_{n}\right\|}\right)}{\left\|x_{n}\right\|}\right]
$$

Here

$$
\lim _{n \rightarrow+\infty} \frac{\varphi\left(\frac{x_{n}}{\left\|x_{n}\right\|}\right)}{\left\|x_{n}\right\|}=0
$$

since the sequence $\frac{x_{n}}{\left\|x_{n}\right\|}$ remains in a compact set and $\varphi$ is continuous. Taking now the limit as $n \rightarrow+\infty$ in (52), we obtain the contradiction $1 \leq 0$. The sequence $\left\{x_{n}\right\}$ is thus bounded.

Setting $v:=x^{*}$ in (50) and $v:=x_{n}$ in (51), we obtain the relations

$$
\left\langle x_{n}-G\left(\lambda_{n}, y_{n}\right), x_{n}-x^{*}\right\rangle-\lambda_{n} \varphi\left(x^{*}\right)+\lambda_{n} \varphi\left(x_{n}\right) \leq 0
$$

and

$$
-\left\langle x^{*}-G\left(\lambda^{*}, y^{*}\right), x_{n}-x^{*}\right\rangle-\lambda^{*} \varphi\left(x_{n}\right)+\lambda^{*} \varphi\left(x^{*}\right) \leq 0 .
$$

Thus

$$
\begin{gathered}
\left\|x_{n}-x^{*}\right\|^{2} \leq\left\|G\left(\lambda_{n}, y_{n}\right)-G\left(\lambda^{*}, y^{*}\right)\right\|\left\|x_{n}-x^{*}\right\| \\
+\left(\lambda_{n}-\lambda^{*}\right) \varphi\left(x^{*}\right)+\left(\lambda^{*}-\lambda_{n}\right) \varphi\left(x_{n}\right) .
\end{gathered}
$$

The sequence $\left\{x_{n}\right\}$ is bounded and thus the sequence $\left\{\varphi\left(x_{n}\right)\right\}$ is bounded too since $\varphi$ is continuous. Moreover $\left\|G\left(\lambda_{n}, y_{n}\right)-G\left(\lambda^{*}, y^{*}\right)\right\| \rightarrow 0$ and $\left(\lambda_{n}-\lambda^{*}\right) \rightarrow 0$ as $n \rightarrow+\infty$. Then (55) yields $x_{n} \rightarrow x^{*}$ as $n \rightarrow+\infty$. 
Proposition 5 Suppose that $F: \mathbb{R}^{n} \rightarrow \mathbb{R}^{n}$ is continuous. Suppose also that there exists $\sigma>0$ and a continuous mapping $H: \mathbb{R}^{n} \rightarrow \mathbb{R}^{n}$ such that
(1) $\Lambda(x) \neq 0, \forall x \in \bar{B}_{\sigma}, x \neq 0$
(2) $\quad H(x) \neq 0, \forall x \in \bar{B}_{\sigma}, x \neq 0$;
(3) $\langle F(x), H(x)\rangle+\varphi(x)-\varphi(x-H(x)) \geq 0, \forall x \in \bar{B}_{\sigma}$.

Then

$$
\zeta(\Lambda, 0)=\operatorname{deg}\left(H, B_{r}, 0\right), \forall r \in(0, \sigma] .
$$

Proof: Let $r \in(0, \sigma]$ be given. Let $h:[0,1] \times \bar{B}_{r} \rightarrow \mathbb{R}^{n} ;(\lambda, y) \rightarrow h(\lambda, y):=y-P_{\lambda \varphi}(y-$ $\lambda F(y)-(1-\lambda) H(y))$. Proposition 4 ensures that $h$ is continuous. Let us now check that $h(\lambda, x) \neq 0, \forall x \in \partial B_{r}, \lambda \in[0,1]$. Indeed, suppose on the contrary that there exists $x \in \mathbb{R}^{n},\|x\|=r$ and $\lambda \in[0,1]$ such that $h(\lambda, x)=0$, that is

$$
x=P_{\lambda \varphi}(x-\lambda F(x)-(1-\lambda) H(x)) .
$$

We first remark that $\lambda \neq 0$. Indeed, suppose that $\lambda=0$. Then $x=P_{0}(x-H(x))=x-H(x)$. This yields $H(x)=0$ which is a contradiction to assumption (2) since here $x \neq 0$. We remark now that $\lambda \neq 1$. Indeed, suppose that $\lambda=1$. Then $x=P_{\varphi}(x-F(x))$ and thus $\Lambda(x)=0$ which is a contradiction to assumption (1) since here $x \neq 0$.

Thus $0<\lambda<1$ and

$$
\langle\lambda F(x)+(1-\lambda) H(x), v-x\rangle+\lambda \varphi(v)-\lambda \varphi(x) \geq 0, \forall v \in \mathbb{R}^{n} .
$$

Setting $v:=x-H(x)$, we obtain

$$
\lambda[\langle F(x), H(x)\rangle+\varphi(x)-\varphi(x-H(x))] \leq-(1-\lambda)\|H(x)\|^{2}<0,
$$

which is a contradiction to assumption (3).

Thus

$$
\begin{aligned}
& \operatorname{deg}\left(i d_{\mathbb{R}^{n}}-P_{\varphi}\left(i d_{\mathbb{R}^{n}}-F\right), B_{r}, 0\right)=\operatorname{deg}\left(h(1, .), B_{r}, 0\right)= \\
& =\operatorname{deg}\left(h(0, .), B_{r}, 0\right)=\operatorname{deg}\left(i d_{\mathbb{R}^{n}}-P_{0}\left(i d_{\mathbb{R}^{n}}-H\right), B_{r}, 0\right)= \\
& =\operatorname{deg}\left(H, B_{r}, 0\right) .
\end{aligned}
$$

INRIA 
Proposition 6 Suppose that $F: \mathbb{R}^{n} \rightarrow \mathbb{R}^{n}$ is continuous. Suppose also that there exists $\sigma>0$ and a continuous mapping $H: \mathbb{R}^{n} \rightarrow \mathbb{R}^{n}$ such that

$$
\langle F(x), H(x)\rangle+\varphi(x)-\varphi(x-H(x))>0, \forall x \in \bar{B}_{\sigma}, x \neq 0 .
$$

Then

$$
\zeta(\Lambda, 0)=\operatorname{deg}\left(H, B_{r}, 0\right), \forall r \in(0, \sigma] .
$$

Proof: The result is a consequence of Proposition 5. It is clear that if (56) holds then conditions (2) and (3) of Proposition 5 are satisfied. We claim that condition (1) of Proposition 5 holds too. Indeed, suppose on the contrary that there exists $x \in \bar{B}_{\sigma} \backslash\{0\}$ such that $\Lambda(x)=0$. Then

$$
\langle F(x), v-x\rangle+\varphi(v)-\varphi(x) \geq 0, \forall v \in \mathbb{R}^{n} .
$$

Setting $v=x-H(x)$, we get

$$
\langle F(x), H(x)\rangle+\varphi(x)-\varphi(x-H(x)) \leq 0 .
$$

which is a contradiction to (56).

\section{The case of linear complementarity problems}

In view of applications like stabilization, we examine in this section the case when the vector field is linear invariant with matrix $A \in \mathbb{R}^{n \times n}$, and $\varphi(\cdot)=\Psi_{K}(\cdot)$ with $K=\mathbb{R}_{+}^{n}$. The following holds:

Proposition 7 Let $F: \mathbb{R}^{n} \rightarrow \mathbb{R}^{n}$ be defined by $F(x)=A x$ with $A \in \mathbb{R}^{n \times n}$ and $\varphi(\cdot)=$ $\Psi_{K}(\cdot)$ with $K=\mathbb{R}_{+}^{n}$. If $A$ satisfies the conditions (i) $A_{i i}>0,1 \leq i \leq n$, and (ii) $\left|\frac{A_{i j}}{A_{i i}}\right|<\frac{1}{n}$ for all $1 \leq i \leq n, 1 \leq j \leq n, i \neq j$, then $\zeta(\lambda, 0)=1$.

Proof: The proof uses Proposition 2. As we have seen in (35), we have $\Lambda_{i}(x)=\min \left\{x_{i}, e_{i}^{T} A x\right\}$, with $e_{i}$ the $i$-th unit vector of $\mathbb{R}^{n}$. Let us now calculate $\Lambda_{i}(x)$ and $\Lambda_{i}(y)$ for the vectors $x$ and $y$ as in (33). Taking into account the definitions of the sets $P_{i}(-\varepsilon)$ and $P_{i}(+\varepsilon), \varepsilon>0$, we obtain

$$
\Lambda_{i}(x)=\min \left\{-\varepsilon, e_{i}^{T} A x+A_{i i}\left(-\varepsilon-x_{i}\right)\right\}
$$

with $x \in[-\varepsilon,+\varepsilon]^{n}$, and

$$
\Lambda_{i}(y)=\min \left\{\varepsilon, e_{i}^{T} A y+A_{i i}\left(\varepsilon-y_{i}\right)\right\}
$$

with $y \in[-\varepsilon,+\varepsilon]^{n}$ (in both these expressions we do not consider the variables $x$ and $y$ in the sets $P_{i}(-\varepsilon)$ and $P_{i}(+\varepsilon)$ respectively, but as free vectors of $\mathbb{R}^{n}$ satisfying $\|x\|_{\infty}(:=$ 
$\left.\max _{1 \leq i \leq n}\left\{\left|x_{i}\right|\right\}\right) \leq \varepsilon$ and $\left.\|y\|_{\infty} \leq \varepsilon\right)$. The condition $\Lambda_{i}(x) \Lambda_{i}(y)<0$ can then be written as

$$
\max \left\{\varepsilon,-e_{i}^{T} A x+A_{i i} x_{i}+A_{i i} \varepsilon\right\} \times \min \left\{\varepsilon, e_{i}^{T} A y-A_{i i} y_{i}+A_{i i} \varepsilon\right\}>0 .
$$

The inequality in (57) is equivalent to

(a) $\max \left\{\varepsilon,-e_{i}^{T} A x+A_{i i} x_{i}+A_{i i} \varepsilon\right\}>0$ and (b) $\min \left\{\varepsilon, e_{i}^{T} A y-A_{i i} y_{i}+A_{i i} \varepsilon\right\}>0$

or

(c) $\max \left\{\varepsilon,-e_{i}^{T} A x+A_{i i} x_{i}+A_{i i} \varepsilon\right\}<0$ and (d) $\min \left\{\varepsilon, e_{i}^{T} A y-A_{i i} y_{i}+A_{i i} \varepsilon\right\}<0$

for all $\|x\|_{\infty} \leq \varepsilon$ and $\|y\|_{\infty} \leq \varepsilon$. The inequality in (c) is impossible to fulfill and the inequality in (a) is always satisfied, since $\varepsilon>0$. We are therefore left with the inequality (b) only, which is satisfied if and only if $e_{i}^{T} A y-A_{i i} y_{i}>-A_{i i} \varepsilon$ for all $y \in \mathbb{R}^{n}$ with $\|y\|_{\infty} \leq \varepsilon$. Using assumption (i), we see that this condition can be rewritten as

$$
\left(e_{i}^{T} \frac{A}{A_{i i}}-e_{i}^{T}\right) y+\varepsilon>0
$$

for all $\|y\|_{\infty} \leq \varepsilon$. Equivalently

$$
\left(e_{i}^{T} \frac{A}{A_{i i}}-e_{i}^{T}\right) y+1>0
$$

for all $\|y\|_{\infty} \leq 1$. It is clear that inequality (59) is satisfied if and only if

$$
\left|\left(e_{i}^{T} \frac{A}{A_{i i}}-e_{i}^{T}\right) y\right|<1, \forall y \in \mathbb{R}^{n},\|y\|_{\infty} \leq 1 .
$$

Assumption (ii) ensures that

$$
\left\|\frac{A}{A_{i i}} e_{i}-e_{i}\right\|_{\infty}=\max _{1 \leq j \leq n}\left|\frac{A_{i j}}{A_{i i}}-\delta_{i j}\right|<1,
$$

where $\delta_{i j}$ is the Kronecker symbol. If $\|y\|_{\infty} \leq 1$ then

$$
\left|\left(e_{i}^{T} \frac{A}{A_{i i}}-e_{i}^{T}\right) y\right| \leq n\left\|\frac{A}{A_{i i}} e_{i}-e_{i}\right\|_{\infty}\|y\|_{\infty} \leq n\left\|\frac{A}{A_{i i}} e_{i}-e_{i}\right\|_{\infty}<1,
$$

so that (60) is satisfied. Finally, Proposition 2 ensures that $\zeta(\Lambda, 0)=1$.

Remark 6 i) From Proposition 3 in [12], it follows that if $A$ is diagonal with positive entries, then the equilibrium $x=0$ is asymptotically stable. Incidentally one sees that $A_{i j}=0, i \neq j$, implies that $\left|\frac{A_{i j}}{A_{i i}}\right|=0<\frac{1}{n}$ so that the necessary conditions for asymptotic stability are satisfied.

ii) The conditions (i) and (ii) are not necessary conditions for asymptotic stability. Indeed matrices $A$ which satisfy ( $i$ ) and $A_{i j} \geq 0$ for all $1 \leq i \leq n, 1 \leq j \leq n$ are strictly copositive on $K$ and guarantee asymptotic stability (see Proposition 3 in [12]).

iii) Condition (ii) implies that $A$ is diagonally dominant.

INRIA 
Example 7 Let us consider a planar case with $A \in \mathbb{R}^{2 \times 2}$. The above conditions read $A_{11}>$ $0, A_{22}>0, \frac{\left|A_{12}\right|}{A_{11}}<\frac{1}{2}$ and $\frac{\left|A_{21}\right|}{A_{22}}<\frac{1}{2}$. We see that $A$ is a P-matrix [9] since both principal minors are positive, as $A_{i i}>0(i=1,2)$ and $\operatorname{det}(A)=A_{11} A_{22}\left(1-\frac{A_{12} A_{21}}{A_{11} A_{22}}\right)>0$. Notice that there exist P-matrices which are not positive definite, like for instance $A=\left(\begin{array}{cc}1 & -3 \\ 0 & 1\end{array}\right)$ [9, p.147]. Also P-matrices may have complex eigenvalues with negative real parts, for instance $A=\left(\begin{array}{ll}1 & -3 \\ 1 & -2\end{array}\right), \lambda_{1}=-\frac{1}{2}+j \frac{\sqrt{3}}{2}, \lambda_{2}=-\frac{1}{2}-j \frac{\sqrt{3}}{2}$. However it can be shown that the above conditions guarantee that the eigenvalues of $A$ have positive real parts. Let $\gamma_{1}$ and $\gamma_{2}$ be the two eigenvalues of $A$. Then $A_{11} A_{22}-A_{12} A_{21}=\gamma_{1} \gamma_{2}$ and $A_{22}+A_{11}=\gamma_{1}+\gamma_{2}$. Since $A$ is a P-matrix, its real eigenvalues are positive. Thus if the eigenvalues have negative real parts, they must be complex conjugate and in such a case $A_{22} A_{11}-A_{12} A_{21}=\gamma_{1} \gamma_{2}>0$ and $A_{22}+A_{11}=\gamma_{1}+\gamma_{2}<0$ so that $A_{22}<-A_{11}$. This is a contradiction since $A_{22}>0$ and $A_{11}>0$. We conclude that in the planar case, the necessary conditions of Proposition 7 imply that $A$ is a P-matrix with (positive) real eigenvalues.

We now make use of Proposition 3 to derive conditions for under which the stationary solution is not asymptotically stable.

Proposition 8 Let $F: \mathbb{R}^{n} \rightarrow \mathbb{R}^{n}$ be defined by $F(x)=A x$ with $A \in \mathbb{R}^{n \times n}$ and $\varphi(\cdot)=$ $\Psi_{K}(\cdot)$ with $K=\mathbb{R}_{+}^{n}$. If $A$ satisfies the conditions (i) $A_{i i}<0,1 \leq i \leq n$, and (ii) $\left|\frac{A_{i j}}{A_{i i}}\right|<\frac{1}{n}$ for all $1 \leq i \leq n, 1 \leq j \leq n, i \neq j$, then $\zeta(\lambda, 0)=0$ and the stationary solution of (19) is not asymptotically stable.

Proof: The proof uses Proposition 3. The calculations are similar to those in the proof of Proposition 7. The conditions in (a), (b), (c), (d) now are

$\left(\mathbf{a}^{\prime}\right) \max \left\{\varepsilon,-e_{i}^{T} A x+A_{i i} x_{i}+A_{i i} \varepsilon\right\}>0$ and $\left(\mathbf{b}^{\prime}\right) \min \left\{\varepsilon, e_{i}^{T} A y-A_{i i} y_{i}+A_{i i} \varepsilon\right\}<0$

or

$$
\left(\mathbf{c}^{\prime}\right) \max \left\{\varepsilon,-e_{i}^{T} A x+A_{i i} x_{i}+A_{i i} \varepsilon\right\}<0 \text { and }\left(\mathbf{d}^{\prime}\right) \min \left\{\varepsilon, e_{i}^{T} A y-A_{i i} y_{i}+A_{i i} \varepsilon\right\}>0
$$

for all $\|x\|_{\infty} \leq \varepsilon$ and $\|y\|_{\infty} \leq \varepsilon$. Inequality ( $\left.\mathbf{c}^{\prime}\right)$ cannot be satisfied since $\varepsilon>0$. Inequality (a') is always satisfied. Inequality (b') is equivalent to having $e_{i}^{T} A y-A_{i i} y_{i}+A_{i i} \varepsilon<0$ for all $\|y\|_{\infty} \leq \varepsilon$. Using assumption (i), this can be rewritten as

$$
\left(e_{i}^{T} \frac{A}{A_{i i}}-e_{i}^{T}\right) y+1>0, \forall y \in \mathbb{R}^{n},\|y\|_{\infty} \leq 1
$$

As in the proof of Proposition 7, we see that Assumption (ii) ensures that (61) holds. We conclude that both (i) and (ii) assure that $\Lambda_{i}(x) \Lambda_{i}(y)>0$ for all $x \in P_{i}(-\varepsilon)$ and $y \in P_{i}(+\varepsilon)$ and all $1 \leq i \leq n$, so that $\zeta(\Lambda, 0)=0$ and the stationary solution of (19) cannot be asymptotically stable. 


\section{Application 1 Asymptotic stabilization}

Let us here consider a controlled system with transition matrix $A \in \mathbb{R}^{n \times n}$, input matrix $B \in \mathbb{R}^{n \times 1}$, and a feedback controller $u()=.U x($.$) with U \in \mathbb{R}^{1 \times n}$. The corresponding mathematical model is of the form given in (19) with $F(x)=(A+B U) x$. Assume that the pair $(A, B)$ has the so-called controller form [31], i.e. $A_{i, i+1}=1$ and $A_{i j}=0$ for all $j \neq i+1$ and all $1 \leq i \leq n-1, A_{n j}=\alpha_{j}$ where the $\alpha_{j}$ 's are the coefficient of the characteristic polynomial of $A$, and $B=e_{n}$. One can show that the closed-loop system does not satisfy the conditions of Proposition 2. Or, that it does satisfy the conditions of Proposition 3. Consequently, a controlled evolution variational inequality as in (19) with $A$ and $B$ in a controller form cannot be asymptotically stabilized by constant feedback. This is a major discrepancy with unconstrained systems. This shows that having $(A, B)$ a controllable pair (for the unconstrained system) is not at all sufficient to guarantee the asymptotic stabilization of a controlled variational inequality. This is in accordance with the results in [8] on controllability of planar variational inequalities.

Conditions ( $i)$ and (ii) of Proposition 7 now read as conditions for asymptotic stabilization: given a pair $(A, B)$, find a matrix $U \in \mathbb{R}^{1 \times n}$ such that

$$
\left\{\begin{array}{l}
A_{i i}+B_{i} U_{i}>0,1 \leq i \leq n \\
\left|A_{i j}+B_{i} U_{j}\right|<\frac{1}{n}\left(A_{i i}+B_{i} U_{i}\right), \quad \forall i \neq j, 1 \leq i \leq n, 1 \leq j \leq n
\end{array}\right.
$$

so that there exists a constant feedback control. We can now set some conditions to be satisfied by $(A, B)$ so that the feedback guarantees $\zeta(\Lambda, 0)=1$ :

- 1) $A \neq 0$ (from (60) (a) and combining with (62) (b)).

- 2) If $A_{i i}=0$ for some $i$, then necessarily $B_{i} \neq 0$ (from (62)(a)).

- 3) If $B_{i}=0$ for some $i$, then necessarily $A_{i i} \neq 0$ (from (62)(a)).

- 4) Controllability of $(A, B)$ (the Kalman matrix is of rank n) is not sufficient.

- 5) Controllability of $(A, B)$ (the Kalman matrix is of rank $n$ ) is not necessary: there exist pairs $(A, B)$ such that the Kalman matrix has rank $<n$, and which satisfy the conditions in (62) or the sufficient condition of Proposition 7.

Item 5) can be proved by choosing a planar example with $A_{11}>0, A_{12}=0, A_{21} \neq 0, A_{22} \neq 0$, and $B_{1}=0, B_{2} \neq 0$. We have $\operatorname{rank}(B \quad A B)=1$, even if $0<\left|A_{21}+B_{2} U_{1}\right|<\frac{1}{2}\left(A_{22}+B_{2} U_{2}\right)$. Let us choose $U_{2}=\operatorname{sgn}\left(B_{2}\right) \frac{\alpha-A_{22}}{\left|B_{2}\right|}, \alpha>0$, and $U_{1}=\operatorname{sgn}\left(B_{2}\right) \frac{\beta-A_{21}}{\left|B_{2}\right|}, \beta \geq 0, \beta<\frac{\alpha}{2}$. Then the sufficient condition of Remark 6 i) is satisfied if $\beta=0$, and conditions (i) are (ii) of Proposition 7 are satisfied if $\beta<\frac{\alpha}{2}$. 


\section{Instability Results}

Inasmuch as stability is usually a desired property, it is important to dispose of some mathematical results which can be used to recognize instability. Here we use Theorem 5 together with Theorem 3 in order to state conditions ensuring instability.

Theorem 8 Suppose that assumptions $\left(h_{1}\right)-\left(h_{7}\right)$ are satisfied. Suppose in addition that there exists $\sigma>0$ and $V \in C^{1}\left(\mathbb{R}^{n} ; \mathbb{R}\right)$ such that

(1) $\varphi()-.\varphi\left(.-V^{\prime}().\right)$ is lower semicontinuous on $D(\partial \varphi) \cap \bar{B}_{\sigma}$;

(2) $\left\langle F(x), V^{\prime}(x)\right\rangle+\varphi(x)-\varphi\left(x-V^{\prime}(x)\right) \geq 0, \forall x \in D(\partial \varphi) \cap \bar{B}_{\sigma}$;

(3) $\mathcal{M}\left(F, \varphi, V, \bar{B}_{\sigma}\right)=\{0\}$,

where (see Section 4) $\mathcal{M}\left(F, \varphi, V, \bar{B}_{\sigma}\right)$ denotes the largest invariant subset of $\mathcal{E}(F, \varphi, V) \cap$ $\bar{B}_{\sigma}=\left\{x \in D(\partial \varphi) \cap \bar{B}_{\sigma}:\left\langle F(x), V^{\prime}(x)\right\rangle+\varphi(x)-\varphi\left(x-V^{\prime}(x)\right)=0\right\}$.

If $\zeta(\Lambda, 0) \neq 1$ then the trivial stationary solution of (19) is unstable.

Proof: Suppose on the contrary that the trivial stationary solution of (19) is stable. Then we may find $\delta>0$ such that if $x_{0} \in \bar{B}_{\delta}$ then $\gamma\left(x_{0}\right) \subset \bar{B}_{\sigma}$. We may apply theorem 3 (with $\left.\Psi:=\bar{B}_{\sigma}\right)$ to get $\lim _{t \rightarrow+\infty} d\left(S(t) x_{0}, \mathcal{M}\left(F, \varphi, V, \bar{B}_{\sigma}\right)\right)=0$. Here $\mathcal{M}\left(F, \varphi, V, \bar{B}_{\sigma}\right)=\{0\}$ and thus the trivial stationary solution of (19) is attractive. It results that the trivial stationary solution of (19) is asymptotically stable and we obtain a contradiction to $\zeta(\Lambda, 0) \neq 1$.

Corollary 2 Suppose that assumptions $\left(h_{1}\right)-\left(h_{7}\right)$ are satisfied. Suppose in addition that there exists $\sigma>0$ and $V \in C^{1}\left(\mathbb{R}^{n} ; \mathbb{R}\right)$ such that

(1) $\varphi()-.\varphi\left(.-V^{\prime}().\right)$ is lower semicontinuous on $D(\partial \varphi) \cap \bar{B}_{\sigma}$;

(2) $\left\langle F(x), V^{\prime}(x)\right\rangle+\varphi(x)-\varphi\left(x-V^{\prime}(x)\right)>0, x \in D(\partial \varphi) \cap \bar{B}_{\sigma}, x \neq 0$.

If $\zeta(\Lambda, 0) \neq 1$ then the trivial stationary solution of (19) is unstable.

Proof: The results is a direct consequence of Theorem 8 since condition (2) ensures that $\mathcal{M}\left(F, \varphi, V, \bar{B}_{\sigma}\right)=\{0\}$.

The following result is a direct consequence of the previous one.

Corollary 3 Let $K \subset \mathbb{R}^{n}$ be a closed convex set such that $0 \in K$. Suppose that assumptions $\left(h_{5}\right)-\left(h_{7}\right)$ are satisfied. Suppose in addition that there exists $\sigma>0$ and $V \in C^{1}\left(\mathbb{R}^{n} ; \mathbb{R}\right)$ such that

$V^{\prime}(x) \in-K_{\infty}, x \in K \cap \bar{B}_{\sigma}$

$\mathrm{RR} \mathrm{n}^{\circ} 5171$ 
$\left\langle F(x), V^{\prime}(x)\right\rangle>0, x \in K \cap \bar{B}_{\sigma}, x \neq 0 ;$

If $\zeta(\Lambda, 0) \neq 1$ then the trivial stationary solution of (19) is unstable.

Example 8 The trivial stationary solution of (19) with the data given in Example 2 (ii) is unstable. Indeed, we know that here $\zeta(\Lambda, 0)=0$ and it is easy to see that the conditions of Corollary 3 hold with $V():.=-\frac{1}{2}\|\cdot\|^{2}$.

Theorem 9 Suppose that $\varphi: \mathbb{R}^{n} \rightarrow \mathbb{R}$ is convex and dom $\{\varphi\}=\mathbb{R}^{n}$. Suppose that assumptions $\left(h_{5}\right)-\left(h_{7}\right)$ are satisfied. Suppose in addition that there exists $\sigma>0$ and $V \in C^{1}\left(\mathbb{R}^{n} ; \mathbb{R}\right)$ such that

(1) $\quad V^{\prime}(x) \neq 0, \forall x \in \bar{B}_{\sigma}, x \neq 0$;

(2) $\left\langle F(x), V^{\prime}(x)\right\rangle+\varphi(x)-\varphi\left(x-V^{\prime}(x)\right) \geq 0, x \in \bar{B}_{\sigma}$;

(3) $\mathcal{M}\left(F, \varphi, V, \bar{B}_{\sigma}\right)=\{0\}$;

(2) $\operatorname{deg}\left(V^{\prime}, B_{r}, 0\right) \neq 1$ for $r>0$ small enough.

Then the trivial stationary solution of (19) is unstable.

Proof: The result is a consequence of Theorem 8 and Proposition 5 with $H:=V^{\prime}$.

Corollary 4 Suppose that $\varphi: \mathbb{R}^{n} \rightarrow \mathbb{R}$ is convex and $\operatorname{dom}\{\varphi\}=\mathbb{R}^{n}$. Suppose that assumptions $\left(h_{5}\right)-\left(h_{7}\right)$ are satisfied. Suppose in addition that there exists $\sigma>0$ and a nonsingular and symmetric matrix $A \in \mathbb{R}^{n \times n}$ such that

$$
\langle F(x), A x\rangle+\varphi(x)-\varphi(x-A x) \geq 0, x \in \bar{B}_{\sigma} .
$$

If $\mathcal{M}\left(F, \varphi, \frac{1}{2}\langle A .,\rangle,. \bar{B}_{\sigma}\right)=\{0\}$ and $\operatorname{det} A<0$ then the trivial stationary solution of (19) is unstable.

Proof: The result is a consequence of Theorem 9 with $V()=.\frac{1}{2}\langle A$., . $\rangle$. Here $\operatorname{deg}\left(V^{\prime}, B_{r}, 0\right)=$ $\operatorname{deg}\left(A, B_{r}, 0\right)=\operatorname{sgn}(\operatorname{det} A)=-1, \forall r>0$.

The following result is a direct consequence of Corollary 4 .

Corollary 5 Suppose that $\varphi: \mathbb{R}^{n} \rightarrow \mathbb{R}$ is convex and dom $\{\varphi\}=\mathbb{R}^{n}$. Suppose that assumptions $\left(h_{5}\right)-\left(h_{7}\right)$ are satisfied. Suppose in addition that there exists $\sigma>0$ and a nonsingular and symmetric matrix $A \in \mathbb{R}^{n \times n}$ such that

$$
\langle F(x), A x\rangle+\varphi(x)-\varphi(x-A x)>0, x \in \bar{B}_{\sigma} \backslash\{0\} .
$$

If $\operatorname{det} A<0$ then the trivial stationary solution of (19) is unstable.

INRIA 
Example 9 Let $F$ and $\varphi$ be defined by

$$
F(x)=\left(\begin{array}{ll}
a & c \\
c & b
\end{array}\right)\left(\begin{array}{l}
x_{1} \\
x_{2}
\end{array}\right)
$$

and

$$
\varphi(x)=d\left|x_{2}\right|,
$$

with $a<0, b>0, c \in \mathbb{R}$ and $d>0$. Setting

$$
A=\left(\begin{array}{rr}
-1 & 0 \\
0 & 1
\end{array}\right)
$$

we see that $\langle F(x), A x\rangle+\varphi(x)-\varphi(x-A x)=-a x_{1}^{2}+b x_{2}^{2}+d\left|x_{2}\right|>0, \forall\left(x_{1}, x_{2}\right) \in \mathbb{R}^{2} \backslash\{(0,0)\}$. Here $\operatorname{det} A=-1$ and thus the trivial stationary solution of (19) is unstable.

\section{Application 2 Second Order Dynamical Systems in Mechanics}

Let us here deal with the following class of second order dynamical systems:

Let $\Phi: \mathbb{R}^{m} \rightarrow \mathbb{R}$ be a convex function with $\operatorname{dom}(\Phi)=\mathbb{R}^{m}$. Let $M, C, K \in \mathbb{R}^{m \times m}$ be given matrices. We consider the problem: Find a function $t \mapsto q(t)(t \geq 0)$ with $q \in$ $C^{1}\left([0,+\infty) ; \mathbb{R}^{m}\right)$, and such that:

$$
\begin{gathered}
\frac{d^{2} q}{d t^{2}} \in L_{\mathrm{loc}}^{\infty}\left(0,+\infty ; \mathbb{R}^{m}\right), \\
\frac{d q}{d t} \text { is right-differentiable on }[0,+\infty), \\
M \frac{d^{2} q}{d t^{2}}(t)+C \frac{d q}{d t}(t)+K q(t) \in-\partial \Phi\left(\frac{d q}{d t}(t)\right) \text {, a.e. } t \geq 0 .
\end{gathered}
$$

The model in (65) can be used in Mechanics to describe the motion of various systems having frictional contact. For such problems, $m$ is the number of degrees of freedom of the system, $M$ is the mass matrix of the system, $C$ is the viscous damping matrix of the system and $K$ is the stiffness matrix. The term $\partial \Phi($.$) is used to model the unilaterality of the contact$ induced by friction forces.

Let us first assume that:

$\left(H_{1}\right) M$ is symmetric and positive definite;

$\left(H_{2}\right) K$ is symmetric and nonsingular;

$\mathrm{RR} \mathrm{n}^{\circ} 5171$ 
$\left(H_{3}\right) x^{T} C x+\Phi(x)-\Phi(0)>0, \forall x \in \mathbb{R}^{m}, x \neq 0$

$\left(H_{4}\right) 0 \in \partial \Phi(0)$.

Let

$$
\sigma(M, K):=\{\lambda \in \mathbb{C}: \operatorname{det}(\lambda M+K)=0\}=\left\{\lambda_{1}, \ldots, \lambda_{m}\right\} .
$$

Using assumptions $\left(H_{1}\right)$ and $\left(H_{2}\right)$, we may assert that (see e.g. [2]):

$$
\sigma(M, K) \subset \mathbb{R},
$$

and there exists a nonsingular matrix $R \in \mathbb{R}^{m \times m}$ such that

$$
R^{T} M R=I \text { and } R^{T} K R=K_{0},
$$

where $I$ denotes the $m \times m$ identity matrix and $K_{0}$ is the diagonal matrix defined by $\left(K_{0}\right)_{i i}=$ $\lambda_{i},(i \in\{1, \ldots, m\}$.

Setting

$$
q:=R z
$$

we may rewrite (65) as follows:

$$
\frac{d^{2} z}{d t^{2}}(t)+R^{T} C R \frac{d z}{d t}(t)+K_{0} z(t) \in-R^{T} \partial \Phi\left(R \frac{d z}{d t}(t)\right), \text { a.e. } t \geq 0 .
$$

Let us here define the convex function $\chi: \mathbb{R}^{m} \rightarrow \mathbb{R}$ by the formula

$$
\chi(w)=(\Phi \circ R)(w), \forall w \in \mathbb{R}^{m} .
$$

Then (see Theorem 23.9 in [26]),

$$
\partial \chi(w)=R^{T} \partial \Phi(R w), \forall w \in \mathbb{R}^{m} .
$$

Let us now set

$$
x_{1}:=z, \quad x_{2}:=\frac{d z}{d t}, \quad x=\left(\begin{array}{ll}
x_{1} & x_{2}
\end{array}\right)^{T}, \quad \dot{x}_{1}=\frac{d x_{1}}{d t}, \dot{x}_{2}=\frac{d x_{2}}{d t} .
$$

It is clear that (66) is equivalent to the following first order system :

$$
\left\{\begin{array}{l}
\dot{x}_{1}-x_{2}=0 \\
\dot{x}_{2}+R^{T} C R x_{2}+K_{0} x_{1} \in-\partial \chi\left(x_{2}\right) .
\end{array}\right.
$$

It results that our problem can be written as in (19) with $n=2 m$ and where $F$ and $\varphi$ are defined by

$$
F(x)=\left(\begin{array}{rr}
0 & -I \\
K_{0} & R^{T} C R
\end{array}\right)\left(\begin{array}{l}
x_{1} \\
x_{2}
\end{array}\right)
$$

and

$$
\varphi(x)=\chi\left(x_{2}\right)
$$


Let $V$ be defined by

$$
V(x)=\frac{1}{2}\langle A x, x\rangle
$$

where

$$
A=\left(\begin{array}{rr}
K_{0} & 0 \\
0 & I
\end{array}\right)
$$

We see that

$$
\begin{gathered}
V^{\prime}(x)=A x \neq 0, \forall x \in \mathbb{R}^{n}, x \neq 0, \\
\operatorname{deg}\left(V^{\prime}, B_{r}, 0\right)=\operatorname{sgn}(\operatorname{det} A)=\operatorname{sgn}\left(\operatorname{det} K_{0}\right), \forall r>0,
\end{gathered}
$$

and

$$
\begin{gathered}
\left\langle F(x), V^{\prime}(x)\right\rangle+\varphi(x)-\varphi\left(x-V^{\prime}(x)\right)=x_{2}^{T} R^{T} C R x_{2}+ \\
+\chi\left(x_{2}\right)-\chi(0) \geq 0, \forall x \in \mathbb{R}^{n} .
\end{gathered}
$$

Here

$$
\mathcal{S}(F, \varphi)=\left\{\left(x_{1}, 0\right): K_{0} x_{1} \in-\partial \chi(0)\right\}
$$

and

$$
\begin{gathered}
\mathcal{E}(F, \varphi, V)=\left\{x \in \mathbb{R}^{n}: x_{2}^{T} R^{T} C R x_{2}+\chi\left(x_{2}\right)-\chi(0)=0\right\}= \\
=\left\{\left(x_{1}, 0\right) ; x_{1} \in \mathbb{R}^{m}\right\} .
\end{gathered}
$$

Moreover, it can be proved that the largest invariant subset $\mathcal{M}(F, \varphi, V)$ of $\mathcal{E}(F, \varphi, V)$ coincides with the set of stationary solutions of (19) (see the proof of Theorem 9 in [1]), that is:

$$
\mathcal{M}(F, \varphi, V)=\mathcal{S}(F, \varphi)
$$

Our theory can be applied provided that the trivial stationary solution of (68) is isolated in $\mathcal{S}(F, \varphi)$. Let us so now assume in place of $\left(H_{4}\right)$ that:

$\left(H_{4}\right)^{\prime} \partial \Phi(0)=\{0\}$.

Then $\mathcal{S}(F, \varphi)=\{0\}$. So, if assumptions $\left(H_{1}\right)-\left(H_{3}\right),\left(H_{4}\right)^{\prime}$ are satisfied, then properties (69)-(71) hold, $\mathcal{M}(F, \varphi, V)=\{0\}$ and we may apply Theorem 9 to conclude that if

$$
\operatorname{det} K_{0}<0
$$

then the trivial stationary solution of (68) is unstable. 


\section{Appendix}

\section{PROOF OF CLAIM 1.}

We first prove that there exists $T^{*}>0$ such that

$$
\left\|S\left(T^{*}\right) P_{D(\partial \varphi)} x\right\| \leq \frac{r}{2}, \forall x \in \bar{B}_{r} .
$$

Indeed, the stability of 0 as a solution of (19) ensures the existence of $\alpha>0$ such that if $\left\|S(\bar{t}) P_{D(\partial \varphi)} x\right\| \leq \alpha$ for some $\bar{t}>0$ then $\left\|S(t) P_{D(\partial \varphi)} x\right\| \leq \frac{r}{2}, \forall t \geq \bar{t}$. Let $X \in \bar{B}_{r}$ be given. The attractivity of 0 as a solution of (19) ensures the existence of $T:=T(X)>0$ such that $\left\|S(T) P_{D(\partial \varphi)} X\right\| \leq \frac{\alpha}{2}$. Setting $\sigma:=\frac{\alpha}{2} \sqrt{e^{-2 \bar{\omega} T}}$, we see that, for $v \in B(X, \sigma):=\left\{z \in \mathbb{R}^{n}\right.$ : $\|z-X\|<\sigma\}$, we have (see (28))

$$
\begin{gathered}
\left\|S(T) P_{D(\partial \varphi)} v\right\| \leq\left\|S(T) P_{D(\partial \varphi)} v-S(T) P_{D(\partial \varphi)} X\right\|+\left\|S(T) P_{D(\partial \varphi)} X\right\| \leq \\
\quad \leq \sqrt{e^{2 \bar{\omega} T}}\left\|P_{D(\partial \varphi)} v-P_{D(\partial \varphi)} X\right\|+\frac{\alpha}{2} \leq \sqrt{e^{2 \bar{\omega} T}}\|v-X\|+\frac{\alpha}{2} \leq \alpha .
\end{gathered}
$$

We see that

$$
\bar{B}_{r} \subset \bigcup_{X \in \bar{B}_{r}} B(X, \sigma)
$$

and since $\bar{B}_{r}$ is compact, we find $X_{1}, X_{2}, \ldots, X_{N}$ ( and corresponding $T_{i}, \sigma_{i}$ ) such that

$$
\bar{B}_{r} \subset \bigcup_{i=1}^{N} B\left(X_{i}, \sigma_{i}\right) .
$$

Setting $T^{*}:=\max \left\{T_{1}, T_{2}, \ldots, T_{N}\right\}$, we see that if $x \in \bar{B}_{r}$ then $x \in B\left(X_{i}, \sigma_{i}\right)$ for some $i \in\{1, \ldots ., N\}$. Then $\left\|S\left(T_{i}\right) P_{D(\partial \varphi)} x\right\| \leq \alpha$ and thus $\left\|S(t) P_{D(\partial \varphi)} x\right\| \leq \frac{r}{2}, \forall t \geq T^{*}\left(\geq T_{i}\right)$.

Let us now check that

$$
\operatorname{deg}\left(i d_{\mathbb{R}^{n}}-S\left(T^{*}\right) P_{D(\partial \varphi) \cdot}, B_{r}, 0\right)=1 .
$$

Let us define the continuous homotopy $h:[0,1] \times \mathbb{R}^{n} \rightarrow \mathbb{R}^{n}$ by

$$
h(\lambda, x):=x-\lambda S\left(T^{*}\right) P_{D(\partial \varphi)} x .
$$

We claim that $h(\lambda, x) \neq 0, \forall \lambda \in[0,1], x \in \partial B_{r}$. Indeed, suppose on the contrary that there exists $x \in \partial B_{r}$ and $\lambda \in[0,1]$ such that

$$
x=\lambda S\left(T^{*}\right) P_{D(\partial \varphi)} x .
$$

Here $x=\lambda S\left(T^{*}\right) P_{D(\partial \varphi)} x+(1-\lambda) 0$ and thus $x \in D(\partial \varphi)$ since $S\left(T^{*}\right) P_{D(\partial \varphi)} x \in D(\partial \varphi)$, $0 \in D(\partial \varphi)$ (by assumption $\left.\left(h_{3}\right)\right), \lambda \in[0,1]$ and $D(\partial \varphi)$ is convex. It results that $x=\lambda S\left(T^{*}\right) x$ and thus

$$
\left\|x-\lambda S\left(T^{*}\right) x\right\|=0 .
$$

INRIA 
However,

$$
\left\|x-\lambda S\left(T^{*}\right) x\right\| \geq\|x\|-\lambda\left\|S\left(T^{*}\right) x\right\| \geq\|x\|-\left\|S\left(T^{*}\right) x\right\| \geq \frac{r}{2}>0,
$$

which is a contradiction. Thus from properties 2 and 3 (Section 2) we get

$$
\begin{gathered}
\operatorname{deg}\left(i d_{\mathbf{R}^{n}}-S\left(T^{*}\right) P_{D(\partial \varphi)}, B_{r}, 0\right)=\operatorname{deg}\left(h(1, .) ., B_{r}, 0\right)= \\
=\operatorname{deg}\left(h(0, .), B_{r}, 0\right)=\operatorname{deg}\left(i d_{\mathbf{R}^{n}}, B_{r}, 0\right)=1 .
\end{gathered}
$$

We end the proof of CLAIM 1 by remarking that

$$
\operatorname{deg}\left(i d_{\mathbb{R}^{n}}-S(t) P_{D(\partial \varphi) \cdot,} B_{r}, 0\right)=\operatorname{deg}\left(i d_{\mathbb{R}^{n}}-S\left(T^{*}\right) P_{D(\partial \varphi)} \cdot, B_{r}, 0\right), \forall t>0 .
$$

Indeed, let $t>0$ be given and define the continuous homotopy $g:[0,1] \times \mathbb{R}^{n} \rightarrow \mathbb{R}^{n}$ by

$$
g(\lambda, x):=x-S\left(\lambda t+(1-\lambda) T^{*}\right) P_{D(\partial \varphi)} x .
$$

We see that $g(\lambda, x) \neq 0, \forall \lambda \in[0,1], x \in \partial B_{r}$. Indeed, suppose on the contrary that there exists $x \in \partial B_{r}$ and $\lambda \in[0,1]$ such that $x=S\left(\lambda t+(1-\lambda) T^{*}\right) P_{D(\partial \varphi)} x$. Then $x \in D(\partial \varphi)$ and thus

$$
x=S\left(\lambda t+(1-\lambda) T^{*}\right) x .
$$

That means that the application $\tau \rightarrow S(\tau) x$ is periodic with period $\lambda t+(1-\lambda) T^{*}$ (see Section 5) and non-trivial since $\|S(0) x\|=\|x\|=r>0$. This is a contradiction to (40). Thus from property 2 in Section 2

$$
\begin{aligned}
& \operatorname{deg}\left(i d_{\mathbf{R}^{n}}-S(t) P_{D(\partial \varphi)} ., B_{r}, 0\right)=\operatorname{deg}\left(g(1, .), B_{r}, 0\right)= \\
= & \operatorname{deg}\left(g(0, .), B_{r}, 0\right)=\operatorname{deg}\left(i d_{\mathbb{R}^{n}}-S\left(T^{*}\right) P_{D(\partial \varphi) .}, B_{r}, 0\right) .
\end{aligned}
$$

\section{PROOF OF CLAIM 2.}

Let $t>0$ be given and define the homotopy $H:[0,1] \times \mathbb{R}^{n} \rightarrow \mathbb{R}^{n}$ by

$$
H(\lambda, x):=x-\left(\lambda i d_{\mathbb{R}^{n}}+t \mathcal{A}_{\varphi}\right)^{-1}\left(\lambda i d_{\mathbb{R}^{n}}+t G\right) P_{D(\partial \varphi)} x,
$$

where $G$ is defined in (42). We first remark that $H$ is continuous. Indeed, let $x_{n} \rightarrow x$ and $\lambda_{n} \rightarrow \lambda$ and set $f_{n}:=\left(\lambda_{n} i d_{\mathbf{R}^{n}}+t G\right) P_{D(\partial \varphi)} x_{n}$ and $v_{n}:=\left(\lambda_{n} i d_{\mathbb{R}^{n}}+t \mathcal{A}_{\varphi}\right)^{-1} f_{n}$. It is clear that

$$
f_{n} \rightarrow f:=\left(\lambda i d_{\mathbb{R}^{n}}+t G\right) P_{D(\partial \varphi)} x .
$$

We claim that

$$
v_{n} \rightarrow v:=\left(\lambda i d_{\mathbf{R}^{n}}+t \mathcal{A}_{\varphi}\right)^{-1} f .
$$


Indeed, we have

$$
\begin{gathered}
v_{n}:=\left(\lambda_{n} i d_{\mathbb{R}^{n}}+t \mathcal{A}_{\varphi}\right)^{-1} f_{n} \Leftrightarrow \frac{f_{n}-\lambda_{n} v_{n}}{t} \in \mathcal{A}_{\varphi} v_{n} \Leftrightarrow \\
\Leftrightarrow\left\langle v_{n}-\left(\frac{f_{n}-\lambda_{n} v_{n}}{t}\right), w-v_{n}\right\rangle+\varphi(w)-\varphi\left(v_{n}\right) \geq 0, \forall w \in \mathbb{R}^{n} .
\end{gathered}
$$

Setting $w=0$ in (72), we get

$$
\left\langle v_{n}-\left(\frac{f_{n}-\lambda_{n} v_{n}}{t}\right), v_{n}\right\rangle \leq \varphi(0)-\varphi\left(v_{n}\right)
$$

and then using (29), we obtain

$$
\begin{gathered}
\left(t+\lambda_{n}\right)\left\|v_{n}\right\|^{2} \leq\left\|f_{n}\right\|\left\|v_{n}\right\|+t\left[\left\langle-F(0), v_{n}\right\rangle+\varphi(0)-\varphi\left(v_{n}\right)\right]+t\left\langle F(0), v_{n}\right\rangle \\
\leq\left\|f_{n}\right\|\left\|v_{n}\right\|+t\|F(0)\|\left\|v_{n}\right\| .
\end{gathered}
$$

It results that the sequence $\left\{v_{n}\right\}$ is bounded.

Setting $w=v$ in (72), we get

$$
\left\langle t v_{n}+\lambda_{n} v_{n}-f_{n}, v-v_{n}\right\rangle+t\left(\varphi(v)-\varphi\left(v_{n}\right)\right) \geq 0 .
$$

We remark also that $v=\left(\lambda i d_{\mathbb{R}^{n}}+t \mathcal{A}_{\varphi}\right)^{-1} f$ if and only if

$$
\left\langle v-\left(\frac{f-\lambda v}{t}\right), w-v\right\rangle+\varphi(w)-\varphi(v) \geq 0, \forall w \in \mathbb{R}^{n},
$$

and thus

$$
\left\langle t v+\lambda v-f, v_{n}-v\right\rangle+t\left(\varphi\left(v_{n}\right)-\varphi(v)\right) \geq 0 .
$$

So, from (73)

$$
\left\langle t v_{n}+\lambda_{n} v_{n}, v_{n}-v\right\rangle \leq t\left(\varphi(v)-\varphi\left(v_{n}\right)\right)+\left\langle f_{n}, v_{n}-v\right\rangle
$$

and from (74)

$$
-\left\langle t v+\lambda v, v_{n}-v\right\rangle \leq t\left(\varphi\left(v_{n}\right)-\varphi(v)\right)-\left\langle f, v_{n}-v\right\rangle .
$$

Thus

$$
\left\langle t\left(v_{n}-v\right)+\lambda_{n} v_{n}-\lambda v, v_{n}-v\right\rangle \leq\left\langle f_{n}-f, v_{n}-v\right\rangle .
$$

We obtain

$$
(t+\lambda)\left\|v_{n}-v\right\|^{2} \leq\left|\lambda-\lambda_{n}\right|\left\|v_{n}\right\|\left\|v_{n}-v\right\|+\left\|f_{n}-f\right\|\left\|v_{n}-v\right\| .
$$

The sequence $\left\{v_{n}\right\}$ is bounded and thus $\left\{v_{n}\right\} \subset \bar{B}_{K}$ for some constant $K>0$. It results that

$$
\left\|v_{n}-v\right\| \leq K\left|\lambda-\lambda_{n}\right|+\left\|f_{n}-f\right\|
$$

INRIA 
and thus $v_{n} \rightarrow v$ as $n \rightarrow \infty$.

We check now that $H(\lambda, x) \neq 0, \forall \lambda \in[0,1], x \in \partial B_{r}$. Suppose on the contrary that there exists $x \in \partial B_{r}$ and $\lambda \in[0,1]$ such that

$$
x=\left(\lambda i d_{\mathbf{R}^{n}}+t \mathcal{A}_{\varphi}\right)^{-1}\left(\lambda i d_{\mathbb{R}^{n}}+t G\right) P_{D(\partial \varphi)} x .
$$

Then

$$
\frac{\lambda\left(P_{D(\partial \varphi)} x-x\right)+t G\left(P_{D(\partial \varphi)} x\right)}{t} \in \mathcal{A}_{\varphi}(x) .
$$

Recalling that $\mathcal{A}_{\varphi}^{-1}=P_{\varphi}$, we get

$$
x=P_{\varphi}\left(\frac{\lambda\left(P_{D(\partial \varphi)} x-x\right)+t G\left(P_{D(\partial \varphi)} x\right)}{t}\right) .
$$

Thus from (8) $x \in D(\partial \varphi)$ and (75) reduces to

$$
x=P_{\varphi}(G(x)),
$$

which is a contradiction to (38).

Then

$$
\begin{gathered}
\operatorname{deg}\left(i d_{\mathbb{R}^{n}}-\left(i d_{\mathbb{R}^{n}}+t \mathcal{A}_{\varphi}\right)^{-1}\left(i d_{\mathbb{R}^{n}}+t G\right) P_{D(\partial \varphi)} \cdot, B_{r}, 0\right)=\operatorname{deg}\left(H(1, .), B_{r}, 0\right)= \\
=\operatorname{deg}\left(H(0, .), B_{r}, 0\right)=\operatorname{deg}\left(i d_{\mathbb{R}^{n}}-\left(t \mathcal{A}_{\varphi}\right)^{-1}\left(t G\left(P_{D(\partial \varphi)} \cdot\right)\right), B_{r}, 0\right)= \\
=\operatorname{deg}\left(i d_{\mathbb{R}^{n}}-P_{\varphi} G\left(P_{D(\partial \varphi)} \cdot\right), B_{r}, 0\right) .
\end{gathered}
$$

\section{PROOF OF CLAIM 3.}

Let us consider the continuous homotopy $\mathcal{G}:[0,1] \times \mathbb{R}^{n} \rightarrow \mathbb{R}^{n}$ by

$$
\mathcal{G}(\lambda, x):=x-P_{\varphi}\left(G\left(\lambda x+(1-\lambda) P_{D(\partial \varphi)} x\right) .\right.
$$

We claim that $\mathcal{G}(\lambda, x) \neq 0, \forall \lambda \in[0,1], x \in \partial B_{r}$. Suppose on the contrary that there exists $x \in \partial B_{r}$ and $\lambda \in[0,1]$ such that

$$
x=P_{\varphi} G\left(\lambda x+(1-\lambda) P_{D(\partial \varphi)} x\right) .
$$

Then $x \in D(\partial \varphi)$ and thus

$$
x=P_{\varphi} G(x),
$$

which is a contradiction to (38). Thus

$$
\begin{gathered}
\operatorname{deg}\left(i d_{\mathbb{R}^{n}}-P_{\varphi} G\left(P_{D(\partial \varphi)} .\right), B_{r}, 0\right)=\operatorname{deg}\left(\mathcal{G}(0, .), B_{r}, 0\right)= \\
=\operatorname{deg}\left(\mathcal{G}(1,0), B_{r}, 0\right)=\operatorname{deg}\left(i d_{\mathbb{R}^{n}}-P_{\varphi} G(.), B_{r}, 0\right) .
\end{gathered}
$$

$\mathrm{RR} \mathrm{n}^{\circ} 5171$ 


\section{PROOF OF CLAIM 4.}

(a) Recalling that $P_{D(\partial \varphi)} 0=0$, we obtain

$$
\left\|P_{D(\partial \varphi)} x\right\|=\left\|P_{D(\partial \varphi)} x-P_{D(\partial \varphi)} 0\right\| \leq\|x\| \leq r \leq r_{0} .
$$

(b) Recalling that $P_{\varphi} G(0)=0$ (see (29) and (42)), we get

$$
\begin{gathered}
\left\|P_{\varphi} G\left(P_{D(\partial \varphi)} x\right)\right\|=\left\|P_{\varphi} G\left(P_{D(\partial \varphi)} x\right)-P_{\varphi} G(0)\right\| \leq\left\|G\left(P_{D(\partial \varphi)} x\right)-G(0)\right\| \leq \\
\leq\left\|P_{D(\partial \varphi)} x\right\|+\left\|F\left(P_{D(\partial \varphi)} x\right)-F(0)\right\| \leq r_{0}\left(1+C_{0}\right) \leq \frac{\delta_{1}}{2}<\delta_{1} .
\end{gathered}
$$

(c) This follows from (a) and (39).

(d) We know that

$$
u(0)=P_{D(\partial \varphi)} x
$$

and

$$
\left\langle\frac{d u}{d s}(s), w-u(s)\right\rangle+\langle F(u(s)), w-u(s)\rangle+\varphi(w)-\varphi(u(s)) \geq 0 \text {, a.e. } s \geq 0 .
$$

Setting $w=P_{D(\partial \varphi)} x$ in (77), we obtain

$$
\begin{gathered}
\left\langle\frac{d}{d s}\left(P_{D(\partial \varphi)} x-u(s)\right), P_{D(\partial \varphi)} x-u(s)\right\rangle \leq \\
\leq\left\langle F(u(s)), P_{D(\partial \varphi)} x-u(s)\right\rangle+\varphi\left(P_{D(\partial \varphi)} x\right)-\varphi(u(s)), \text { a.e. } s \geq 0 .
\end{gathered}
$$

Using (a), (c) and (37), we obtain

$$
\varphi\left(P_{D(\partial \varphi)} x\right)-\varphi(u(s)) \leq C_{1}\left\|P_{D(\partial \varphi)} x-u(s)\right\| \leq C_{1}\left(r_{0}+1\right) .
$$

We have also

$$
\begin{gathered}
\left\langle F(u(s)), P_{D(\partial \varphi)} x-u(s)\right\rangle= \\
=\left\langle F(u(s))-F\left(P_{D(\partial \varphi)} x\right), P_{D(\partial \varphi)} x-u(s)\right\rangle+\left\langle F\left(P_{D(\partial \varphi)} x\right), P_{D(\partial \varphi)} x-u(s)\right\rangle \leq \\
\leq \bar{\omega}\left\|P_{D(\partial \varphi)} x-u(s)\right\|^{2}+\left\|F\left(P_{D(\partial \varphi)} x\right)\right\|\left\|P_{D(\partial \varphi)} x-u(s)\right\| \leq \\
\leq \bar{\omega}\left(r_{0}+1\right)^{2}+\left(r_{0}+1\right)\left(\left\|F\left(P_{D(\partial \varphi)} x\right)-F(0)\right\|+\|F(0)\|\right) \leq \\
\leq \bar{\omega}\left(r_{0}+1\right)^{2}+\left(r_{0}+1\right)\left(C_{0} r_{0}+\|F(0)\|\right) .
\end{gathered}
$$


Thus

$$
\frac{1}{2} \frac{d}{d s}\left\|P_{D(\partial \varphi)} x-u(s)\right\|^{2} \leq\left(r_{0}+1\right)\left(C_{1}+\bar{\omega}\left(r_{0}+1\right)+C_{0} r_{0}+\|F(0)\|\right) \text {, a.e. } s \geq 0 .
$$

Consequently, for $t>0$, we have

$$
\int_{0}^{t} \frac{d}{d s}\left\|P_{D(\partial \varphi)} x-u(s)\right\|^{2} d s \leq 2\left(r_{0}+1\right)\left(C_{1}+\bar{\omega}\left(r_{0}+1\right)+C_{0} r_{0}+\|F(0)\|\right) t .
$$

Using now (76), we obtain

$$
\left\|P_{D(\partial \varphi)} x-u(t)\right\| \leq \theta \sqrt{t} .
$$

(e) Using (c) and recalling that $P_{\varphi} G(0)=0$, we get

$$
\begin{gathered}
\left\|P_{\varphi} G(u(t))\right\|=\left\|P_{\varphi} G(u(t))-P_{\varphi} G(0)\right\| \leq \\
\leq\|G(u(t))-G(0)\| \leq\|u(t)\|+\|F(u(t))-F(0)\| \leq \\
\leq\|u(t)\|\left(1+C_{0}\right) \leq \frac{\delta_{1}}{2}<\delta_{1} .
\end{gathered}
$$

(f) Let $t \in\left(0, t^{*}\right]$ be given. Using Theorem 4 , we obtain

$$
\left\|S\left(t^{*}\right) P_{D(\partial \varphi)} x-S\left(t^{*}\right) 0\right\| \leq \sqrt{e^{2 w\left(t^{*}-t\right)}}\left\|S(t) P_{D(\partial \varphi)} x-S(t) 0\right\| .
$$

Thus

$$
\|u(t)\| \geq \sqrt{e^{2 \bar{\omega} t}} \sqrt{e^{-2 \bar{\omega} t^{*}}}\left\|u\left(t^{*}\right)\right\| \geq \sqrt{e^{-2 \bar{\omega} t^{*}}} \frac{r}{4} \geq \frac{r}{8} .
$$

(g) From the definition of $v(t)$, we see that

$$
\frac{P_{D(\partial \varphi)} x-v(t)+t G\left(P_{D(\partial \varphi)} x\right)}{t} \in \mathcal{A}_{\varphi}(v(t)) .
$$

Thus

$$
\begin{gathered}
\left\langle\frac{v(t)-P_{D(\partial \varphi)} x}{t}, w-v(t)\right\rangle+\left\langle v(t)-G\left(P_{D(\partial \varphi)} x\right), w-v(t)\right\rangle \\
+\varphi(w)-\varphi(v(t)) \geq 0, \forall w \in \mathbb{R}^{n} .
\end{gathered}
$$

It results also that

$$
\frac{v(t)-P_{D(\partial \varphi)} x}{t}+v(t)-G\left(P_{D(\partial \varphi)} x\right) \in-\partial \varphi(v(t))
$$

and thus $v(t) \in D(\partial \varphi)$.

$\mathrm{RR} \mathrm{n}^{\circ} 5171$ 
(h) Applying the result in (g) with $w:=P_{D(\partial \varphi)} x$, we obtain

$$
\begin{gathered}
\frac{1}{t}\left\langle v(t)-P_{D(\partial \varphi)} x, P_{D(\partial \varphi)} x-v(t)\right\rangle+\left\langle v(t)-G\left(P_{D(\partial \varphi)} x\right), P_{D(\partial \varphi)} x-v(t)\right\rangle+ \\
+\varphi\left(P_{D(\partial \varphi)} x\right)-\varphi(v(t)) \geq 0 .
\end{gathered}
$$

Thus

$$
\begin{gathered}
\frac{1}{t}\left\|v(t)-P_{D(\partial \varphi)} x\right\|^{2}+\left\|v(t)-P_{D(\partial \varphi)} x\right\|^{2} \leq \\
\leq\left\langle P_{D(\partial \varphi)} x-G\left(P_{D(\partial \varphi)} x\right), P_{D(\partial \varphi)} x-v(t)\right\rangle+\varphi\left(P_{D(\partial \varphi)} x\right)-\varphi(v(t))= \\
=\left\langle F\left(P_{D(\partial \varphi)} x\right), P_{D(\partial \varphi)} x-v(t)\right\rangle+\varphi\left(P_{D(\partial \varphi)} x\right)-\varphi(v(t))= \\
=\left\langle F\left(P_{D(\partial \varphi)} x\right)-F(0), P_{D(\partial \varphi)} x-v(t)\right\rangle+\left\langle F(0), P_{D(\partial \varphi)} x\right\rangle-\langle F(0), v(t)\rangle+ \\
+\varphi(0)-\varphi(v(t))+\varphi\left(P_{D(\partial \varphi)} x\right)-\varphi(0) \leq \\
\leq\left\|F\left(P_{D(\partial \varphi)} x\right)-F(0)\right\|\left\|P_{D(\partial \varphi)} x-v(t)\right\|+\|F(0)\|\left\|P_{D(\partial \varphi)} x\right\|+\left|\varphi\left(P_{D(\partial \varphi)} x\right)-\varphi(0)\right| \leq \\
\leq C_{0} r_{0}\left\|P_{D(\partial \varphi)} x-v(t)\right\|+\|F(0)\| r_{0}+C_{1} r_{0} \leq \\
\leq \frac{\left(C_{0} r_{0}\right)^{2}}{2}+\frac{\left\|P_{D(\partial \varphi)} x-v(t)\right\|^{2}}{2}+\|F(0)\| r_{0}+C_{1} r_{0} .
\end{gathered}
$$

Thus

$$
\frac{1}{t}\left\|v(t)-P_{D(\partial \varphi)} x\right\|^{2} \leq \frac{\left(C_{0} r_{0}\right)^{2}}{2}+\|F(0)\| r_{0}+C_{1} r_{0} .
$$

Therefore

$$
\left\|v(t)-P_{D(\partial \varphi)} x\right\| \leq \bar{\theta} \sqrt{t} .
$$

(i) Let $\mathrm{t} \in\left(0, T^{*}\right]$ be given. From (a) and (h), we deduce that

$$
\|v(t)\| \leq \bar{\theta} \sqrt{t}+r_{0} .
$$

Thus

$$
\|v(t)\| \leq \frac{\delta_{0}}{2}+r_{0} \leq \delta_{0}
$$

and

$$
\|v(t)\| \leq \frac{\delta_{1}}{2\left(1+C_{0}\right)}+r_{0} \leq \frac{\delta_{1}}{1+C_{0}} .
$$

(j) Let $\mathrm{t} \in\left(0, T^{*}\right]$ be given. Using (i), we get

$$
\begin{gathered}
\| P_{\varphi}\left(G ( v ( t ) ) \| = \| P _ { \varphi } \left(G(v(t))-P_{\varphi}(G(0))\|\leq\| G(v(t))-G(0)\|\leq\| v(t)\|+\| F(v(t))-F(0) \| \leq\right.\right. \\
\leq\left(1+C_{0}\right)\|v(t)\| \leq \delta_{1} .
\end{gathered}
$$

INRIA 


\section{PROOF OF CLAIM 5.}

Let us define the homotopy $W:[0,1] \times \mathbb{R}^{n} \rightarrow \mathbb{R}^{n}$ by

$$
W(\lambda, x):=x-\lambda\left(i d_{\mathbb{R}^{n}}+t \mathcal{A}_{\varphi}\right)^{-1}\left(i d_{\mathbb{R}^{n}}+t G\right) P_{D(\partial \varphi)} x-(1-\lambda) S(t) P_{D(\partial \varphi)} x .
$$

Note that $W(\lambda, x)=x-\lambda H(1, x)-(1-\lambda) S(t) P_{D(\partial \varphi)} x$ where $H$ is defined in Claim 2. The continuity of $W$ follows from the the continuity of the Poincare operator $S(t)$. and the continuity of $H$ (see Claim 2). We claim that $W(\lambda, x) \neq 0, \forall \lambda \in[0,1], x \in \partial B_{r}$. Indeed, suppose on the contrary that there exists $x \in \partial B_{r}$ and $\lambda \in[0,1]$ such that

$$
x=\lambda\left(i d_{\mathbb{R}^{n}}+t \mathcal{A}_{\varphi}\right)^{-1}\left(i d_{\mathbb{R}^{n}}+t G\right) P_{D(\partial \varphi)} x+(1-\lambda) S(t) P_{D(\partial \varphi)} x .
$$

Setting

$$
u(t):=S(t) P_{D(\partial \varphi)} x
$$

and

$$
v(t):=\left(i d_{\mathbb{R}^{n}}+t \mathcal{A}_{\varphi}\right)^{-1}\left(i d_{\mathbb{R}^{n}}+t G\right) P_{D(\partial \varphi)} x,
$$

we may write

$$
x=\lambda v(t)+(1-\lambda) u(t) .
$$

The following calculations will show that this equality is impossible to satisfy on the time interval $(0, \bar{T}]$. Here $u(t) \in D(\partial \varphi)$ and $v(t) \in D(\partial \varphi)$ (see Claim $4(\mathrm{~g})$ ). Thus $x \in D(\partial \varphi)$ since $D(\partial \varphi)$ is convex. It results that

$$
P_{D(\partial \varphi)} x=x .
$$

Moreover, from Claim 1 and Claim 2, we deduce that $\lambda \in(0,1)$. We know that

$$
\begin{gathered}
\left\langle\frac{d u}{d s}(s), w-u(s)\right\rangle+\langle F(u(s)), w-u(s)\rangle+ \\
+\varphi(w)-\varphi(u(s)) \geq 0, \forall w \in \mathbb{R}^{n}, \text { a.e. } s \geq 0,
\end{gathered}
$$

and thus

$$
\begin{gathered}
\int_{0}^{t}\left\langle\frac{d u}{d s}(s), w-u(s)\right\rangle d s+\int_{0}^{t} \varphi(w) d s-\int_{0}^{t} \varphi(u(s)) d s \geq \\
\geq-\int_{0}^{t}\langle F(u(s)), w-u(s)\rangle d s, \forall w \in \mathbb{R}^{n} .
\end{gathered}
$$

On the other hand

$$
\int_{0}^{t}\left\langle\frac{d u}{d s}(s), u(s)-x\right\rangle d s=\int_{0}^{t}\left\langle\frac{d}{d s}(u(s)-x), u(s)-x\right\rangle d s=
$$

$\mathrm{RR} \mathrm{n}^{\circ} 5171$ 


$$
=\int_{0}^{t} \frac{d}{d s}\|u(s)-x\|^{2} d s=\frac{1}{2}\|u(t)-x\|^{2}-\frac{1}{2}\|u(0)-x\|^{2}=\frac{1}{2}\|u(t)-x\|^{2} \geq 0 .
$$

Thus

$$
\begin{gathered}
\int_{0}^{t}\left\langle\frac{d u}{d s}(s), w-x\right\rangle d s \geq \int_{0}^{t} \varphi(u(s)) d s+ \\
-\int_{0}^{t} \varphi(w) d s-\int_{0}^{t}\langle u(s)-G(u(s)), w-u(s)\rangle d s, \forall w \in \mathbb{R}^{n} .
\end{gathered}
$$

Let us set

$$
\Xi(t):=\int_{0}^{t}\left\langle\frac{d u}{d s}(s), P_{\varphi}(G(x))-x\right\rangle d s .
$$

Setting $w:=P_{\varphi}(G(x))$ in (79), we see that

$$
\begin{gathered}
\Xi(t) \geq \int_{0}^{t} \varphi(u(s)) d s-\int_{0}^{t} \varphi\left(P_{\varphi}(G(x))\right) d s-\int_{0}^{t}\left\langle u(s)-G(u(s)), P_{\varphi}(G(x))-u(s)\right\rangle d s \\
=\int_{0}^{t} \varphi(u(s)) d s-\int_{0}^{t} \varphi\left(P_{\varphi}(G(x))\right) d s-\int_{0}^{t}\left\langle u(s)-G(u(s)), P_{\varphi}(G(u(s)))-u(s)\right\rangle d s \\
-\int_{0}^{t}\left\langle u(s)-G(u(s)), P_{\varphi}(G(x))-P_{\varphi}(G(u(s)))\right\rangle d s .
\end{gathered}
$$

By definition of $P_{\varphi}$, we have

$$
\left\langle P_{\varphi}(G(u(s)))-G(u(s)), w-P_{\varphi}(G(u(s)))\right\rangle+\varphi(w)-\varphi\left(P_{\varphi}(G(u(s)))\right) \geq 0, \forall w \in \mathbb{R}^{n} .
$$

Setting $w:=u(s)$, we get

$$
\begin{gathered}
\left\langle P_{\varphi}(G(u(s))), G(u(s))\right\rangle \geq-\left\langle P_{\varphi}(G(u(s))), u(s)\right\rangle+\langle G(u(s)), u(s)\rangle+ \\
+\left\|P_{\varphi}(G(u(s)))\right\|^{2}+\varphi\left(P_{\varphi}(G(u(s)))\right)-\varphi(u(s)) .
\end{gathered}
$$

Thus

$$
\begin{gathered}
\varphi(u(s))-\varphi\left(P_{\varphi}(G(x))\right)+\left\langle u(s)-G(u(s)), u(s)-P_{\varphi}(G(u(s)))\right\rangle= \\
=\varphi(u(s))-\varphi\left(P_{\varphi}(G(x))\right)+\|u(s)\|^{2}+\left\langle G(u(s)), P_{\varphi}(G(u(s)))\right\rangle+ \\
-\langle G(u(s)), u(s)\rangle-\left\langle u(s), P_{\varphi}(G(u(s)))\right\rangle \geq \\
\geq \varphi\left(P_{\varphi}(G(u(s)))\right)-\varphi\left(P_{\varphi}(G(x))\right)+\|u(s)\|^{2}-2\left\langle P_{\varphi}(G(u(s))), u(s)\right\rangle+\left\|P_{\varphi}(G(u(s)))\right\|^{2}= \\
=\varphi\left(P_{\varphi}(G(u(s)))\right)-\varphi\left(P_{\varphi}(G(x))\right)+\left\|u(s)-P_{\varphi}(G(u(s)))\right\|^{2} .
\end{gathered}
$$

Thus

$$
\Xi(t) \geq \int_{0}^{t} \varphi\left(P_{\varphi}(G(u(s)))\right)-\varphi\left(P_{\varphi}(G(x))\right)+\left\|u(s)-P_{\varphi}(G(u(s)))\right\|^{2} d s
$$

INRIA 


$$
-\int_{0}^{t}\left\langle u(s)-G(u(s)), P_{\varphi}(G(x))-P_{\varphi}(G(u(s)))\right\rangle d s .
$$

Using Claim 4 (e) and (b), (a), (c) and (d), we see that

$$
\begin{gathered}
\varphi\left(P_{\varphi}(G(u(s)))\right)-\varphi\left(P_{\varphi}(G(x))\right) \leq C_{1}\left\|P_{\varphi}(G(u(s)))-P_{\varphi}(G(x))\right\| \leq \\
\leq C_{1}\|G(u(s))-G(x)\| \leq C_{1}\|u(s)-x\|+C_{1}\|F(u(s))-F(x)\| \leq \theta\left(C_{1}+C_{1} C_{0}\right) \sqrt{s} .
\end{gathered}
$$

Using Claim 4 (c), (a) and (d), we obtain also

$$
\begin{gathered}
\left\langle u(s)-G(u(s)), P_{\varphi}(G(x))-P_{\varphi}(G(u(s)))\right\rangle \leq\|F(u(s))\|\left\|P_{\varphi}(G(x))-P_{\varphi}(G(u(s)))\right\| \leq \\
\leq\left(\|F(0)\|+C_{0}\|u(s)\|\right)\|G(x)-G(u(s))\| \leq \\
\leq\left(\|F(0)\|+C_{0}\right)(\|x-u(s)\|+\|F(x)-F(u(s))\|) \leq \\
\leq\left(\|F(0)\|+C_{0}\right)\left(1+C_{0}\right)\|x-u(s)\| \leq\left(\|F(0)\|+C_{0}\right)\left(1+C_{0}\right) \theta \sqrt{s} .
\end{gathered}
$$

Thus

$$
\Xi(t) \geq \int_{0}^{t}\left\|u(s)-P_{\varphi}(G(u(s)))\right\|^{2} d s-\frac{2}{3} \theta\left(1+C_{0}\right)\left(\|F(0)\|+C_{0}+C_{1}\right) t^{\frac{3}{2}} .
$$

If $\|u(t)\| \geq \frac{r}{4}$ then using Claim 4 (f),we may assert that $\|u(s)\| \geq \frac{r}{8}, \forall s \in(0, t]$ and then using (41), we obtain that

$$
\Xi(t) \geq \eta^{2} t-C t^{\frac{3}{2}} \text { if }\|u(t)\| \geq \frac{r}{4}
$$

It is also clear that

$$
\Xi(t) \geq-C t^{\frac{3}{2}} \text { if }\|u(t)\|<\frac{r}{4}
$$

By Claim 4 (g), we know that

$$
\begin{gathered}
\left\langle\frac{v(t)-x}{t}, w-v(t)\right\rangle+\langle v(t)-G(x), w-v(t)\rangle+ \\
+\varphi(w)-\varphi(v(t)) \geq 0, \forall w \in \mathbb{R}^{n} .
\end{gathered}
$$

Moreover,

$$
\begin{gathered}
\left\langle\frac{v(t)-x}{t}, w-v(t)\right\rangle=\left\langle\frac{v(t)-x}{t}, w-x\right\rangle+\left\langle\frac{v(t)-x}{t}, x-v(t)\right\rangle \leq \\
\leq\left\langle\frac{v(t)-x}{t}, w-x\right\rangle=-\frac{(1-\lambda)}{\lambda t}\langle u(t)-x, w-x\rangle \\
=-\frac{(1-\lambda)}{\lambda t} \int_{0}^{t}\left\langle\frac{d u}{d s}(s), w-x\right\rangle d s .
\end{gathered}
$$

RR $\mathrm{n}^{\circ} 5171$ 
Setting $\nu:=\frac{(1-\lambda)}{\lambda}$, we see that

$$
\begin{gathered}
-\frac{\nu}{t} \int_{0}^{t}\left\langle\frac{d u}{d s}(s), w-x\right\rangle d s+\langle v(t)-G(x), w-v(t)\rangle+ \\
+\varphi(w)-\varphi(v(t)) \geq 0, \forall w \in \mathbb{R}^{n} .
\end{gathered}
$$

Setting $w:=P_{\varphi}(G(x))$, we get

$$
\begin{gathered}
\Xi(t) \leq \frac{t}{\nu}\left[\left\langle v(t)-G(x), P_{\varphi}(G(x))-v(t)\right\rangle+\varphi\left(P_{\varphi}(G(x))\right)-\varphi(v(t))\right]= \\
=\frac{t}{\nu}\left[\left\langle v(t)-G(v(t)), P_{\varphi}(G(x))-v(t)\right\rangle+\left\langle G(v(t))-G(x), P_{\varphi}(G(x))-v(t)\right\rangle+\right. \\
\left.+\varphi\left(P_{\varphi}(G(x))\right)-\varphi(v(t))\right]= \\
=\frac{t}{\nu}\left[\left\langle v(t)-G(v(t)), P_{\varphi}(G(v(t)))-v(t)\right\rangle+\varphi\left(P_{\varphi}(G(v(t)))\right)-\varphi(v(t))+\right. \\
+\left\langle v(t)-G(v(t)), P_{\varphi}(G(x))-P_{\varphi}(G(v(t)))\right\rangle+\left\langle G(v(t))-G(x), P_{\varphi}(G(x))-v(t)\right\rangle+ \\
\left.+\varphi\left(P_{\varphi}(G(x))\right)-\varphi\left(P_{\varphi}(G(v(t)))\right)\right] .
\end{gathered}
$$

We know that

$$
\left\langle P_{\varphi}(G(v(t)))-G(v(t)), w-P_{\varphi}(G(v(t)))\right\rangle+\varphi(w)-\varphi\left(P_{\varphi}(G(v(t)))\right) \geq 0, \forall w \in \mathbb{R}^{n} .
$$

Setting $w:=v(t)$, we see that

$$
\left\langle P_{\varphi}(G(v(t)))-G(v(t)), P_{\varphi}(G(v(t)))-v\right\rangle+\varphi\left(P_{\varphi}(G(v(t)))\right)-\varphi(v(t)) \leq 0
$$

and thus

$$
\begin{gathered}
\left\langle v(t)-G(v(t)), P_{\varphi}(G(v(t)))-v(t)\right\rangle+\varphi\left(P_{\varphi}(G(v(t)))\right)-\varphi(v(t)) \leq \\
\leq-\left\|P_{\varphi}(G(v(t)))-v(t)\right\|^{2} .
\end{gathered}
$$

Thus

$$
\begin{gathered}
\Xi(t) \leq-\frac{t}{\nu}\left\|P_{\varphi}(G(v(t)))-v(t)\right\|^{2}+ \\
+\frac{t}{\nu}\left[\left\langle v(t)-G(v(t)), P_{\varphi}(G(x))-P_{\varphi}(G(v(t)))\right\rangle+\left\langle G(v(t))-G(x), P_{\varphi}(G(x))-v(t)\right\rangle+\right. \\
\left.+\varphi\left(P_{\varphi}(G(x))\right)-\varphi\left(P_{\varphi}(G(v(t)))\right)\right] .
\end{gathered}
$$

Using Claim 4 (a), (h) and (i), we see that

$$
\begin{gathered}
\left\langle v(t)-G(v(t)), P_{\varphi}(G(x))-P_{\varphi}(G(v(t)))\right\rangle \leq\|F(v(t))\|\|G(x)-G(v(t))\| \leq \\
\leq\left(\|F(0)\|+C_{0} \delta_{0}\right)(\|x-v(t)\|+\|F(x)-F(v(t))\|) \leq
\end{gathered}
$$

INRIA 


$$
\leq\left(\|F(0)\|+C_{0} \delta_{0}\right)\left(1+C_{0}\right) \bar{\theta} \sqrt{t} .
$$

Using Claim 4 (a), (b) and (i), we obtain also that

$$
\begin{aligned}
\left\langle G(v(t))-G(x), P_{\varphi}(G(x))-v(t)\right\rangle & \leq\left(\left\|P_{\varphi}(G(x))\right\|+\|v(t)\|\right)(\|x-v(t)\|+ \\
+\|F(x)-F(v(t))\|) & \leq\left(\delta_{1}+\delta_{0}\right)\left(1+C_{0}\right) \bar{\theta} \sqrt{t} .
\end{aligned}
$$

Moreover, using Claim 4 (b), (g), (a), (i) and (h), we get

$$
\begin{gathered}
\left|\varphi\left(P_{\varphi}(G(x))\right)-\varphi\left(P_{\varphi}(G(v(t)))\right)\right| \leq C_{1}\left\|P_{\varphi}(G(x))-P_{\varphi}(G(v(t)))\right\| \leq \\
\leq C_{1}\|G(x)-G(v(t))\| \leq C_{1}\left(1+C_{0}\right)\|x-v(t)\| \leq C_{1}\left(1+C_{0}\right) \bar{\theta} \sqrt{t} .
\end{gathered}
$$

Thus

$$
\begin{aligned}
\Xi(t) \leq-\frac{t}{\nu} \| P_{\varphi}(G(v(t))) & -v(t) \|^{2}+\frac{1}{\nu} t^{\frac{3}{2}}\left[\bar{\theta}\left(1+C_{0}\right)\left(\|F(0)\|+\delta_{1}+\left(1+C_{0}\right) \delta_{0}+C_{1}\right)\right] \\
& \leq-\frac{t}{\nu}\left\|P_{\varphi}(G(v(t)))-v(t)\right\|^{2}+\frac{C}{\nu} t^{\frac{3}{2}}
\end{aligned}
$$

Thus

$$
\Xi(t) \leq-\eta^{2} \frac{t}{\nu}+\frac{C}{\nu} t^{\frac{3}{2}} \text { if }\|v(t)\| \geq \frac{r}{8}
$$

and

$$
\Xi(t) \leq \frac{C}{\nu} t^{\frac{3}{2}} \text { if }\|v(t)\|<\frac{r}{8} .
$$

If $\|u(t)\| \geq \frac{r}{4}$ and $\|v(t)\| \geq \frac{r}{8}$ then from (80) and (82), we get

$$
\eta^{2} t-C t^{\frac{3}{2}} \leq-\eta^{2} \frac{t}{\nu}+\frac{C}{\nu} t^{\frac{3}{2}}
$$

and thus $t \geq \frac{\eta^{4}}{C^{2}}$ which is a contradiction since $t \leq \bar{T} \leq \frac{1}{2} \frac{\eta^{4}}{C^{2}}$.

If $\|u(t)\|<\frac{r}{4}$ and $\|v(t)\| \geq \frac{r}{8}$ then from (81) and (82), we get

$$
-C t^{\frac{3}{2}} \leq-\eta^{2} \frac{t}{\nu}+\frac{C}{\nu} t^{\frac{3}{2}}
$$

Using Claim 4 (i), we see that

$$
\nu=\frac{\|v(t)-x\|}{\|x-u(t)\|} \leq \frac{\|v(t)\|+\|x\|}{\|x\|-\|u(t)\|} \leq \nu^{*}
$$

where $\nu^{*}=\frac{4}{3} \frac{\left(\delta_{0}+r\right)}{r}$. Here (84) yields $t \geq \frac{\eta^{4}}{C^{2}\left(\nu^{*}+1\right)^{2}}$ which contradicts $t \leq \bar{T}$. 
If $\|u(t)\| \geq \frac{r}{4}$ and $\|v(t)\|<\frac{r}{8}$ then from (80) and (83), we get

$$
\eta^{2} t-C t^{\frac{3}{2}} \leq \frac{C}{\nu} t^{\frac{3}{2}}
$$

Using Claim 4 (c), we see that

$$
\nu=\frac{\|v(t)-x\|}{\|x-u(t)\|} \geq \frac{\|x\|-\|v(t)\|}{\|x\|+\|u(t)\|} \geq \nu_{*},
$$

where $\nu_{*}=\frac{7 r}{8(1+r)}$. Then (85) gives $t \geq \frac{\eta^{4}}{C^{2}\left(\frac{1}{\nu_{*}}+1\right)^{2}}$ and a contradiction to $t \leq \bar{T}$.

If $\|u(t)\|<\frac{r}{4}$ and $\|v(t)\|<\frac{r}{8}$ then

$$
\|x\| \leq \lambda\|v(t)\|+(1-\lambda)\|u(t)\| \leq \lambda \frac{r}{8}+(1-\lambda) \frac{r}{4}<r
$$

which is a contradiction since $x \in \partial B_{r}$.

Consequently one has $x \neq \lambda v(t)+(1-\lambda) u(t), \forall x \in \partial B_{r}, \lambda \in[0,1]$ so that we have proved $W(\lambda, x) \neq 0, \forall x \in \partial B_{r}, \lambda \in[0,1]$ Thus

$$
\begin{gathered}
\operatorname{deg}\left(i d_{\mathbb{R}^{n}}-\left(i d_{\mathbb{R}^{n}}+t \mathcal{A}_{\varphi}\right)^{-1}\left(i d_{\mathbb{R}^{n}}+t G\right) P_{D(\partial \varphi)} ., B_{r}, 0\right)=\operatorname{deg}\left(W(1, .), B_{r}, 0\right)= \\
=\operatorname{deg}\left(W(0, .), B_{r}, 0\right)=\operatorname{deg}\left(i d_{\mathbb{R}^{n}}-S(t) P_{D(\partial \varphi) .}, B_{r}, 0\right) .
\end{gathered}
$$

\section{References}

[1] S. Adly and D. Goeleven, A Stability Theory for Second-Order Nonsmooth Dynamical Systems with Applications to Friction Problems, Journal de Mathématiques Pures et Appliquées 83 (2004), 17-51.

[2] R. Bellman, Introduction to Matrix Analysis, McGraw-Hill, New York, 1973.

[3] H. Brézis, Problèmes Unilatéraux, J. Math. Pures Appl. 51, 1-168, 1972.

[4] B. Brogliato, Absolute Stability and the Lagrange-Dirichlet Theorem with Monotone Multivalued Mappings, Systems and Control Letters 51, 343-353, 2004.

[5] B. Brogliato, Some Perspectives on the Analysis and Control of Complementarity Systems, IEEE Transactions on Automatic Control, 48, 918-935, 2003.

[6] B. Brogliato, Nonsmooth Mechanics, Springer Verlag London, 1999.

[7] B. Brogliato and D. Goeleven, The Krakovskii-LaSalle Invariance Principle for a Class of Unilateral Dynamical Systems, submitted to Math. of Control, Signals and Systems.

INRIA 
[8] B. Brogliato, Some results on the controllability of planar variational inequalities, INRIA Research Report no 4788, http://www.inria.fr/rrrt/index.en.html, submitted to Systems and Control Letters.

[9] R.W. Cottle, J.S. Pang, R.E. Stone, The Linear Complementarity Problem, Academic Press, Computer Science and Scientific Computing, 1992.

[10] M. Frémond, Non-Smooth Thermomechanics, Springer Verlag, Berlin-Heidelberg, 2002.

[11] Ch. Glocker, Set Valued Force Laws, Springer, Lecture Notes in Applied Mechanics, LNAM 1, 2001.

[12] D. Goeleven, B. Brogliato, Stability and Instability matrices for Linear Evolution Variational Inequalities, to appear in IEEE Transactions on Automatic Control, 49, 521-534, 2004.

[13] D. Goeleven, D. Motreanu, Y. Dumont, M. Rochdi, Variational and Hemivariational Inequalities - Theory, Methods and Applications. Vol.1: Unilateral Analysis and Unilateral Mechanics, Kluwer Academic Publishers, Boston, 2003.

[14] D. Goeleven, D. Motreanu, Variational and Hemivariational Inequalities - Theory, Methods and Applications. Vol.2: Unilateral Problems and Unilateral Mechanics, Kluwer Academic Publishers, Boston, 2003.

[15] D. Goeleven, M. Motreanu, V. Motreanu, On the Stability of Stationary Solutions of Evolution Variational Inequalities, Advances in Nonlinear Variational Inequalities, 6, 1-30, 2003.

[16] W.P.M.H. Heemels, S. Weiland, Linear Complementarity Systems, SIAM J. Applied Math., 60, 1234-1269, 2000.

[17] N. Kikuchi, J.T. Oden, Contact Problems in Elasticity. A Study of Variational Inequalities and Finite Element Methods, SIAM Publ., Philadelphia, 1988.

[18] N.G. Lloyd, Degree Theory, Cambridge University Press 1978.

[19] J. Mawhin, Continuation Theorems and Periodic Solutions of Ordinary Differential Equations, in Topological Methods in Differential Equations and Inclusions (ed. A. Granas and M. Frigon), NATO ASI Series, Mathematical and Physical Sciences, Vol. 472, Kluwer Academic Publishers, 1994.

[20] A.N. Michel, B. Hu, Towards a Stability Theory of General Hybrid Systems, Automatica, 35, 371-384, 1999.

[21] A.N. Michel, K. Wang, B. Hu, Qualitative Theory of Dynamical Systems, 2nd Edition, Marcel Dekker, Pure and Applied Mathematics, New York, 2001. 
[22] A. Nagurney, D. Zhang Projected Dynamical Systems in the Formulation, Stability Analysis, and Computation of Fixed-Demand Traffic Network Equilibria, Transportation Science, 31, 147-158, 1997.

[23] P. Quittner, On the Principle of Linearized Stability for Variational Inequalities, Math. Ann., 283, 257-270, 1989.

[24] P. Quittner, On the Stability of Stationary Solutions of Parabolic Variational Inequalities, Czech. Math. Journal, 40, 472-474, 1990.

[25] P. Quittner, An Instability Criterion for Variational Inequalities, Nonlinear Analysis, T.M.A. 15, 1167-1180, 1990.

[26] R.T. Rockafellar, Convex Analysis, Princeton University Press, Princeton, New Jersey, 1970.

[27] N. Rouche and J. Mawhin, Equations Différentielles Ordinaires, Tome 2, Masson and Cie, Paris, 1973.

[28] N. Rouche, P. Habets, M. Laloy, Stability Theory by Liapunov's Direct Method, Springer Verlag, New York, 1977.

[29] A. van der Schaft, H. Schumacher, An Introduction to Hybrid Dynamical Systems, Springer, London, LNCIS 251, 2000.

[30] R.E. Showalter, Monotone Operators in Banach Spaces and Nonlinear Partial Differential Equations, American Mathematical Society, USA, 1997.

[31] E.D. Sontag, Mathematical Control Theory; Deterministic Finite Dimensional Systems, Springer Texts in Applied Mathematics 6, second edition, 1998.

[32] A. Szulkin, Positive Solutions of Variational Inequalities: A Degree Theoretic Approach, Journal of Differential Equations, 57, 90-111, 1985.

[33] A.A. Ten Dam, E. Dwarshuis, J.C. Willems, The Contact Problem for Linear Continuous-Time Dynamical Systems: A Geometric Approach, IEEE Transactions on Automatic Control, 42, 458-472, April, 1997.

[34] M. Vidyasagar, Nonlinear Systems Analysis, second edition, Prentice Hall International Editions, 1993.

\section{Contents}

1 Introduction 3

2 Preliminaries on Topological Degree Theory 5 
3 Generalized Projection Operator and its Inverse 6

4 Unilateral Dynamical Systems $\quad 8$

5 The Poincaré Operator $\quad 11$

6 Necessary Conditions of Asymptotic Stability 14

7 The case $\varphi=\psi_{K}$ whith $K$ closed and convex 21

8 The case $\varphi$ convex and $\operatorname{dom}\{\varphi\}=\mathbb{R}^{n} \quad \mathbf{2 4}$

9 The case of linear complementarity problems $\quad 27$

10 Instability Results 31

11 Appendix $\quad 36$

$\mathrm{RR} \mathrm{n}^{\circ} 5171$ 


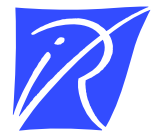

Unité de recherche INRIA Rhône-Alpes

655, avenue de l'Europe - 38330 Montbonnot-St-Martin (France)

Unité de recherche INRIA Futurs : Domaine de Voluceau - Rocquencourt - BP 105 - 78153 Le Chesnay Cedex (France) Unité de recherche INRIA Lorraine : LORIA, Technopôle de Nancy-Brabois - Campus scientifique 615, rue du Jardin Botanique - BP 101 - 54602 Villers-lès-Nancy Cedex (France)

Unité de recherche INRIA Rennes : IRISA, Campus universitaire de Beaulieu - 35042 Rennes Cedex (France) Unité de recherche INRIA Rocquencourt : Domaine de Voluceau - Rocquencourt - BP 105 - 78153 Le Chesnay Cedex (France) Unité de recherche INRIA Sophia Antipolis : 2004, route des Lucioles - BP 93 - 06902 Sophia Antipolis Cedex (France) 